NOTICE: this is the author's version of a work that was accepted for publication in Journal of sound and vibration. Changes resulting from the publishing process, such as peer review, editing, corrections, structural formatting, and other quality control mechanisms may not be reflected in this document. Changes may have been made to this work since it was submitted for publication. A definitive version was subsequently published in Journal of sound and vibration, [VOL 331, ISSUE 22, (10/2012)] DOI 10.1016/j.jsv.2012.06.001

\title{
The finite layer method for modelling the sound transmission through double walls
}

\author{
C. Díaz-Cereceda*1 ${ }^{* 1}$ J. Poblet-Puig ${ }^{\dagger 1}$ and A. Rodríguez-Ferran ${ }^{\ddagger 1}$ \\ ${ }^{1}$ Laboratori de Càlcul Numèric, E.T.S. d'Enginyers de Camins, Canals \\ i Ports de Barcelona, Universitat Politècnica de Catalunya
}

\begin{abstract}
The finite layer method (FLM) is presented as a discretisation technique for the computation of noise transmission through double walls. It combines a finite element method (FEM) discretisation in the direction perpendicular to the wall with trigonometric functions in the two in-plane directions. It is used for solving the Helmholtz equation at the cavity inside the double wall, while the wall leaves are modelled with the thin plate equation and solved with modal analysis. Other approaches to this problem are described here (and adapted where needed) in order to compare them with the FLM. They range from impedance models of the double wall behaviour to different numerical methods for solving the Helmholtz equation in the cavity. For the examples simulated in this work (impact noise and airborne sound transmission), the former are less accurate than the latter at low frequencies. The main advantage of FLM over the other discretisation techniques is the possibility of extending it to multilayered structures without changing the interpolation functions and with an affordable computational cost. This potential is illustrated with a calculation of the noise transmission through a multilayered structure: a double wall partially filled with absorbing material.
\end{abstract}

\section{Introduction}

Lightweight structures are increasingly used in construction, in order to cheaply provide load-bearing configurations with good acoustic properties and a minimal mass. One of the most important structural elements in lightweight structures is double walls: they consist of two leaves with an air cavity (which might be totally or partially filled with absorbing material) between them.

Due to the increasing use of these elements, there is interest in reliable models of their sound insulation. These models should reproduce the acoustic behaviour of

\footnotetext{
*e-mail:cristina.diaz.cereceda@gmail.com

${ }^{\dagger}$ correspondence: UPC, Campus Nord B1, Jordi Girona 1, E-08034 Barcelona, Spain, e-mail: jordi.poblet@upc.edu

${ }^{\ddagger} \mathrm{e}$-mail: antonio.rodriguez-ferran@upc.edu
} 
the double walls both for impact and airborne noise. They should also cover a wide frequency range $(50-5000 \mathrm{~Hz})$ in order to evaluate the outputs defined in regulations $[2,1]$, such as the weighted sound reduction index $R_{w}$.

The main objective of this paper is to present the potential of the finite layer method as a numerical technique for modelling the acoustic behaviour of double walls.

A review of models of sound transmission through double walls is done by Hongisto in [26]. Most of these models assume the structure as infinite and use the impedance approach for the propagation of sound in the cavity. Examples of these models are those suggested by Beranek [9], London [30] and Fahy [21].

Another type of models, more complex than the previous ones, are wave models. Kropp and Rebillard [29] use a wave approach to the problem, with different behaviours depending on the frequency: when the double wall resonance frequency is much lower than the critical frequency, the double wall acts as a single plate; when it is much higher than the critical frequency, the wall leaves act as independent plates; and for resonance frequencies close to the critical frequency, the wall has an intermediate behaviour. Wang et al. [43] express the incident waves, the pressure field inside the cavity and the radiated waves in terms of their velocity potentials. Guigou-Carter and Villot [25] show a wave model that also takes into account the structural flanking paths with the help of statistical energy analysis (SEA).

Some authors have developed impedance models for multilayered structures. They also assume an infinite size for the leaves and express the transmission of pressure and vibrations with the help of transfer matrices. Geebelen et al. [24] consider layers of poroelastic materials inside and Dijckmans et al. [20] compare the results of the transfer matrix method with those of a wave-based model. Brouard et al. [10] combine transfer matrices with interface matrices in order to take into account the interfaces between layers.

Other authors solve a one-dimensional version of the problem with the help of numerical techniques. Trochidis and Kalaroutis [39] use Fourier transforms and Alba et al. [5] refine this technique using an iterative method.

To account for the wall size and boundary conditions, different techniques are employed. Numerical methods can be used for solving the thin plate equation at the wall leaves and the Helmholtz equation at the cavity. Beyond the use of FEM, whose cost happens to be extremely high [33], other discretisation techniques can be applied.

One of them is the use of modal bases for solving the differential equations, as done by Sjökvist et al. [36], Brunskog [11] and Chung and Emms [17]. It consists of expressing the vibration and acoustic fields in terms of the eigenfunctions of the problem. This approach has less computational cost than FEM but is restricted to simple geometries, since the eigenfunctions of complex domains cannot be obtained analytically. However, the typical shapes of leaves and cavities fulfill this requirement. The analysis performed in this work is focused on the cavity path of sound transmission. Thus, for simplicity and clarity, stiffening elements such as frames or ribs have not been considered. However, they could be modelled with the technique described in [19] or, in wave-based models, with the approach used by Vigran [41].

Numerical methods are also used by Xin et al. [45]. They describe the behaviour of the pressure field with the sound velocity potential and express this potential in terms of the modal functions of the leaves. 
Wave-based models usually consider infinite structures radiating in unbounded acoustic domains. However, the finite dimensions of the structures can be considered improving these models. Villot et al. [42] present a technique that introduces the diffraction effect associated to the finite size of a structure using spatial windowing. Kernen and Hassan [28] also emphasise the treatment of finite dimension structures.

Another approach is the waveguide finite element method (WFEM) [32, 4, 12]. This technique, particularly suitable for structures where one dimension is clearly larger than the others, is based on the FEM discretisation of the cross-section of the structure, combined with a wave description of the vibration and pressure fields in the third dimension. It is particularly useful for long structures with complex crosssections. The wave length of the solution is imposed along the extrusion direction in most of the cases. This is not the case of the double wall cavity where the FLM is better adapted to the geometry of the problem.

An approach similar to the WFEM is the finite strip method (FSM) [16, 22]. It also combines the finite element discretisation of the cross-section of the structure with a wave-like description in the third dimension. The main difference with the WFEM is that the FSM considers a finite size of the structure in the third dimension, accounting for the corresponding boundary conditions.

In this work, the finite layer method [37, 15] is proposed as an alternative way of discretising the pressure field in the cavity inside double walls. To the best of the authors' knowledge, this is the first time a finite layer method is proposed for solving the Helmholtz equation. This method combines a FEM-like discretisation in the direction perpendicular to the wall with trigonometric functions in the two inplane directions. The idea is similar to that of the finite strip method but this one is particularly suitable for multilayered structures because it allows the resolution of the Helmholtz equation at each layer, taking into account the continuity of the normal velocity at their interfaces. Moreover, due to the use of trigonometric functions in the in-plane directions, its computational cost is significantly lower than that of pure finite element analysis [34].

The main contribution of this paper is the development of a finite layer numerical model for airborne and impact sound transmission of double walls that accounts for:

- Finite dimensions of the leaves and cavity.

- Realistic boundary conditions: simply supported boundary for the leaves and reflecting cavity contour, see Fig. 1.

- Continuity of the normal velocity at the cavity-leaf interfaces.

- Simple extension to layered double walls.

- Affordable computational cost at high frequencies, in comparison to FEM.

This approach is verified by comparing it with experimental data. It is also compared with existing prediction models in order to check the influence of common simplifications. Some of these simplifications are: use of interpolation functions with null derivative in the boundary, assumption of infinite size of the wall, etc. 
LEAF A

CAVITY

LEAF B

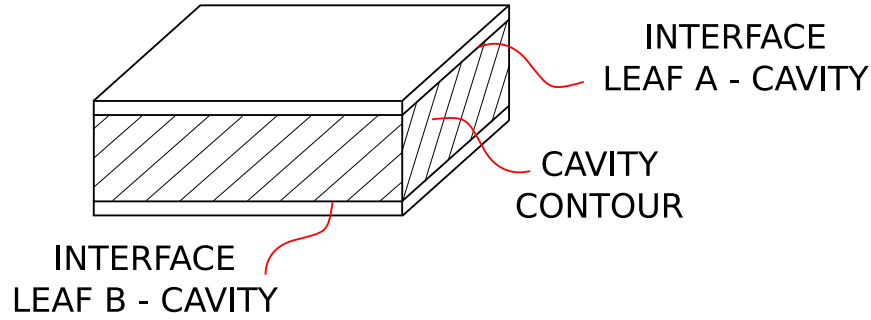

Figure 1: Sketches of the double wall and its parts.

Energy-based models like SEA are not considered in this work. There are different ways of treating double walls with them [27], and the discussion about the different SEA approaches for double walls is beyond the scope of this work. These topics will be analysed in a forthcoming contribution.

An outline of the paper follows. A review of the models for double walls used in this paper is done in Section 2. Then, the basis of the finite layer method applied to double walls is presented in Section 3. Comparisons with experiments and between the models are shown in Section 4, for different excitations and structural configurations. A discussion of the results is provided in Section 5 and the conclusions of Section 6 close the paper.

\section{Models of double walls}

The approach presented in this work is compared with other models of sound transmission through double walls, which illustrate different approaches to the problem. First, two simple impedance models are considered, developed by Fahy [21] and Au and Byrne [7] respectively.

The third approach is a technique based on the model of Xin et al. [45]. This technique does less assumptions than the impedance models but still has less degrees of freedom than the pure numerical approaches. It uses the same interpolation functions for the velocity potential and the plate displacement.

Finally an approach based on the discretisation of the wall leaves and cavity in terms of their separate eigenfunctions is analysed. This description is more realistic than the others. However, the functions used for discretising the pressure field have a null value of the normal derivative at the fluid-structure interface. Due to this, the continuity of the normal velocity at the interfaces can only be enforced in a weak form.

\subsection{Simple impedance equations}

The first two techniques are impedance models that assume infinite size of the wall. They provide explicit expressions for the transmission loss of the double walls for a certain incidence angle, $\tau(\varphi)$. These approaches have almost no computational cost but include a large amount of simplifications. They are based on the $2 \mathrm{D}$ sketch of Fig. 2. 


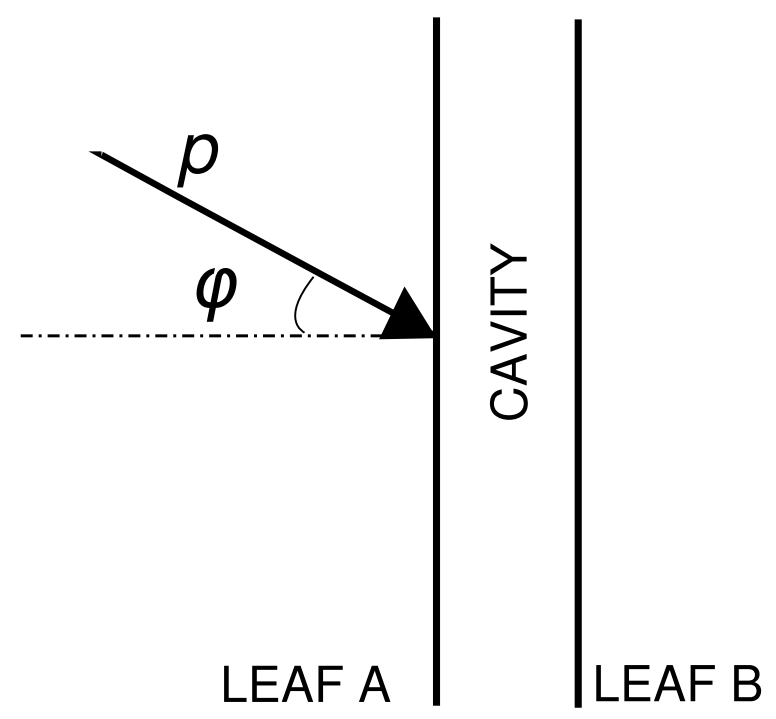

Figure 2: Angle of incidence of the pressure.

In order to obtain regulated outputs such as the sound reduction index $R$, the transmission loss is computed for several incidence angles and averaged. In this way a field incidence is simulated

$$
\tau_{\text {diff }}=\frac{\int_{0}^{\varphi_{\lim }} \tau(\varphi) \cos (\varphi) \sin (\varphi) \mathrm{d} \varphi}{\int_{0}^{\varphi_{\lim }} \cos (\varphi) \sin (\varphi) \mathrm{d} \varphi}
$$

In all the examples the value of $\varphi_{\lim }$ is chosen equal to $78^{\circ}$ following the recommendations of Beranek [8] for a diffuse field.

The sound reduction index is computed as

$$
R=10 \log _{10}\left(\frac{1}{\tau_{\text {diff }}}\right) .
$$

\subsubsection{Fahy 1985}

Fahy presented in [21] a model for empty cavity and diffuse sound incidence angle. In this model, the transmission loss is computed in terms of the mechanical impedances of the leaves and cavity, following a one-dimensional analysis of the problem and taking the lowest eigenfrequency of the leaves into account. The main limitation of this model is that it does not consider the resonant transmission.

The expression for the transmission loss depending on the incidence angle, $\tau(\varphi)$, is shown in A.

\subsection{2 $\mathrm{Au}$ and Byrne 1987}

The second impedance model is the one presented by Au and Byrne [7]. This model assumes that the wave number component parallel to the leaf surface is the same in all of the layers. It also enforces the continuity of the acoustical pressure and particle velocity at the interfaces. It distinguishes between the input impedance $Z_{I}$, 
the terminal impedance $Z_{T}$ and the specific impedance $Z_{i}$ of each material layer $i$, and the calculation starts from the receiving room side. The formulation is shown in B.

\subsection{Adaptation of model of Xin et al. 2010}

A more complex approach is presented in the paper by Xin et al. [45]. This technique consists on solving the thin plate equation for the leaves and describing the pressure field in the cavity with the sound velocity potential method.

The thin plate equation

$$
D \nabla^{4} u(x, y)-\omega^{2} \rho_{s} u(x, y)=q(x, y)+p^{\mathrm{int}}(x, y),
$$

is solved with the boundary conditions of a simply supported plate

$$
\begin{gathered}
u(0, y)=u\left(L_{x}, y\right)=u(x, 0)=u\left(x, L_{y}\right)=0 \\
M(0, y)=M\left(L_{x}, y\right)=M(x, 0)=M\left(x, L_{y}\right)=0 .
\end{gathered}
$$

In Eq. (3), $D=E h^{3} / 12\left(1-\nu^{2}\right)$ is the bending stiffness of the leaf (with $h, E$ and $\nu$ the thickness, Young's modulus and Poisson's ratio of the leaf respectively), $\rho_{s}$ its mass per unit surface, $\omega=2 \pi f$ (with $f$ the frequency of vibration) and $u(x, y)$ the displacement of the leaf. The term $q(x, y)$ is the applied excitation pressure and $p^{\text {int }}(x, y)$ is the pressure of the cavity fluid at the leaf-cavity interface. In Eq. (4), $M(x, y)$ is the bending moment and $L_{x}$ and $L_{y}$ are the dimensions of the leaf, see Fig. 3.

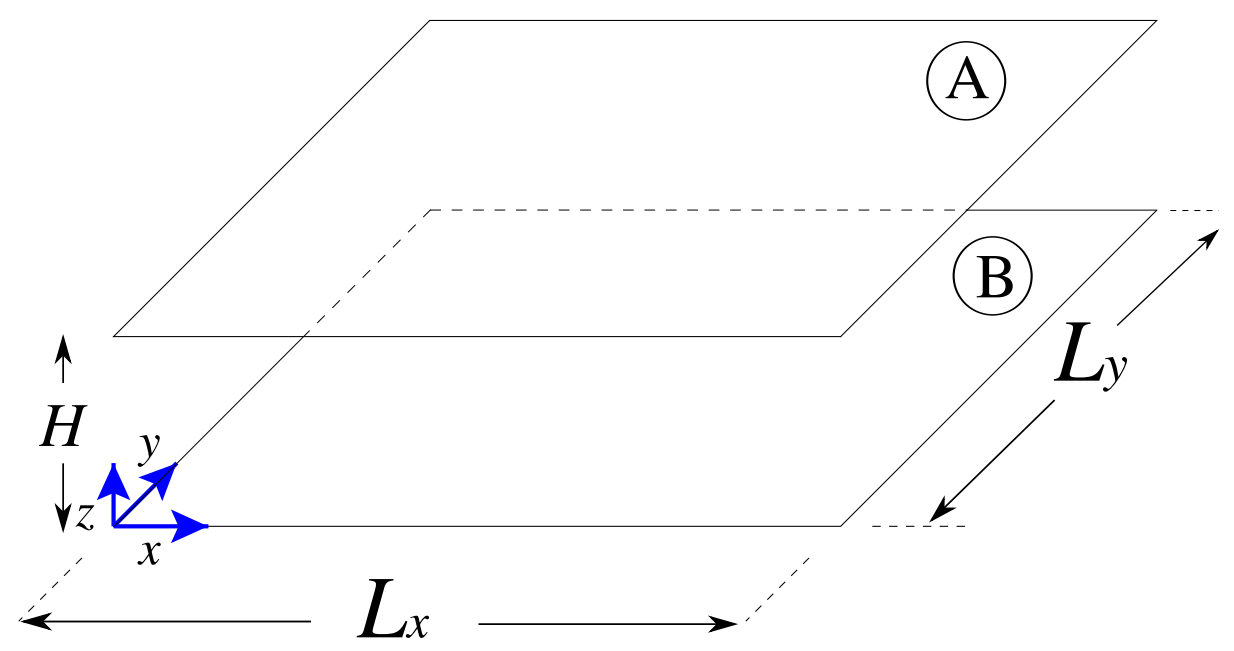

Figure 3: Double wall dimensions.

The vibration field solution of Eq. (3) is expressed in terms of the eigenfunctions $\phi_{r}$ of a simply supported plate as

$$
u(x, y)=\sum_{r=1}^{n_{\text {modes }}} a_{r} \phi_{r}(x, y)
$$


where $n_{\text {modes }}$ is the number of modal functions used in the interpolation, $a_{r}$ is the phasor modal contribution of mode $\phi_{r}$ and

$$
\phi_{r}=\sin \left(\frac{r_{x} \pi x}{L_{x}}\right) \sin \left(\frac{r_{y} \pi y}{L_{y}}\right), \quad r_{x}, r_{y}=1,2, \ldots
$$

The pressure field inside the cavity is expressed in terms of the velocity potential $\Psi(\mathbf{x})$ as

$$
p(\mathbf{x})=\mathrm{i} \omega \rho \Psi(\mathbf{x})
$$

In Eq. (7), $\mathrm{i}=\sqrt{-1}, \mathbf{x}=(x, y, z), \rho$ is the air density and

$$
\Psi(\mathbf{x})=\sum_{r=1}^{n_{\text {modes }}} \epsilon_{r} \phi_{r}(x, y) \exp \left(-\mathrm{i} k_{z} z\right)+\sum_{r=1}^{n_{\text {modes }}} \zeta_{r} \phi_{r}(x, y) \exp \left(\mathrm{i} k_{z} z\right) .
$$

Here, $k_{z}=k \cos \varphi, k=\omega / c$ is the wavenumber in the air and $c$ is the speed of sound in the air.

Xin et al. [45] use this description of the pressure field both for the pressure inside the cavity and for the incident and radiated pressures. However, in this work the velocity potential is only used for the pressure field inside the cavity. The computation of the incident and radiated pressures is performed as described in Section 3.3, allowing a better comparison with the other techniques.

For each frequency and couple of modes $\left\{r_{x}, r_{y}\right\}, \epsilon_{r}$ and $\zeta_{r}$ are expressed in terms of the vibration of the plates by means of the leaf-cavity interface conditions

$$
\begin{array}{ll}
\frac{\partial \Psi}{\partial z}=\mathrm{i} \omega u_{A} & \text { at } z=H \\
\frac{\partial \Psi}{\partial z}=\mathrm{i} \omega u_{B} & \text { at } z=0
\end{array}
$$

In Eq. (9) $u_{A}(x, y)=\sum_{r=1}^{n_{\text {modes }}} a_{r} \phi_{r}(x, y)$ is the vibration field in the excited leaf of the wall, $u_{B}(x, y)=\sum_{r=1}^{n_{\text {modes }}} b_{r} \phi_{r}(x, y)$ is the vibration field in the other one and $H$ is the thickness of the cavity.

Hence, after replacing the expression (7) of the pressure field $p(\mathbf{x})$ in the differential equations of the leaves (3), for each frequency and couple of modes $\left\{r_{x}, r_{y}\right\}$ a 2 degreeof-freedom system has to be solved with $a_{r}$ and $b_{r}$ as unknowns.

The main drawback of this approach is that the description of the pressure field implies a null value of the pressure at the cavity contour. This condition is somehow restrictive, since the contour of finite-dimension cavities is usually purely reflecting with boundary condition:

$$
\nabla p \cdot \mathbf{n}=0
$$

or absorbing with low values of absorption. In both cases a cosine description of the pressure field in the cavity is more adequate than a sine based description, Eq. (6). In Eq. (10), $\mathbf{n}$ is the outward unit normal. The effect of these conditions is discussed in Section 4. 


\subsection{Discretisation with modal bases}

The boundary conditions (4) and (10) can be taken into account if the thin plate equation (3) for each leaf and the Helmholtz equation

$$
\nabla^{2} p(\mathbf{x})+k^{2} p(\mathbf{x})=0
$$

for the acoustic domain are solved numerically with the help of a discretisation of the domain.

This discretisation can be performed in different ways. Here, the separate eigenfunctions of the acoustic and structural equations are used as interpolation bases for the pressure and vibration fields respectively.

In this model, the discretisation of the vibration field in the leaves is the same as in Section 2.2. The integral form of the thin plate equation is used for each leaf

$$
\int_{\Omega_{x y}}\left(D k_{r}^{4}-\omega^{2} \rho_{s}\right) u v \mathrm{~d} x \mathrm{~d} y=\int_{\Omega_{x y}}\left(q+p^{\mathrm{int}}\right) v \mathrm{~d} x \mathrm{~d} y \quad \forall v,
$$

where $v$ is the test function, $\Omega_{x y}=\left[0, L_{x}\right] \times\left[0, L_{y}\right]$ is the leaf domain and

$$
k_{r}^{2}=\left(\frac{r_{x} \pi}{L_{x}}\right)^{2}+\left(\frac{r_{y} \pi}{L_{y}}\right)^{2} .
$$

The pressure field is expressed through a modal expansion

$$
p(\mathbf{x})=\sum_{s=1}^{n_{\text {modes }}} p_{s} \psi_{s}(\mathbf{x})
$$

where $n_{\text {modes }}$ is the number of modes considered for the interpolation. The cavity eigenfunctions are

$$
\psi_{s}(\mathbf{x})=\cos \left(\frac{s_{x} \pi x}{L_{x}}\right) \cos \left(\frac{s_{y} \pi y}{L_{y}}\right) \cos \left(\frac{s_{z} \pi z}{H}\right) \quad s_{x}, s_{y}, s_{z}=0,1,2 \ldots
$$

as if the whole cavity boundary was reflecting [40].

This modal expansion is replaced in the integral version of the Helmholtz equation

$$
\begin{aligned}
\int_{\Omega_{c}} p \nabla^{2} v \mathrm{~d} \Omega+k^{2} \int_{\Omega_{c}} p v \mathrm{~d} \Omega & +\int_{\Omega_{x y}} \rho \omega^{2} v(x, y, H)\left(\mathbf{u}_{\mathbf{A}} \cdot \mathbf{n}\right) \mathrm{d} x \mathrm{~d} y+ \\
& +\int_{\Omega_{x y}} \rho \omega^{2} v(x, y, 0)\left(\mathbf{u}_{\mathbf{B}} \cdot \mathbf{n}\right) \mathrm{d} x \mathrm{~d} y=0 \quad \forall v
\end{aligned}
$$

where $\Omega_{c}=\left[0, L_{x}\right] \times\left[0, L_{y}\right] \times[0, H]$ is the cavity domain, $\mathbf{u}_{\mathbf{A}}=\left(0,0, u_{A}\right)$ and $\mathbf{u}_{\mathbf{B}}=\left(0,0, u_{B}\right)$ are the vibration fields in leaves $\mathrm{A}$ and $\mathrm{B}$ respectively.

All the eigenfunctions of the cavity used in the interpolation are also used as test functions. The coupling with the leaves is imposed in the third and fourth terms of Eq. (16), connecting the modal contributions of the pressure field with those of the vibration fields of the leaves. 
If the only external excitation is applied to leaf $\mathrm{A}$, the resulting block tridiagonal linear system is

$$
\left(\begin{array}{ccc}
\mathrm{A} & \mathrm{A}_{\mathrm{C}} & 0 \\
\mathrm{C}_{\mathrm{A}} & \mathrm{C} & \mathrm{C}_{\mathrm{B}} \\
0 & \mathrm{~B}_{\mathrm{C}} & \mathrm{B}
\end{array}\right)\left\{\begin{array}{l}
\mathrm{a} \\
\mathrm{p} \\
\mathrm{b}
\end{array}\right\}=\left\{\begin{array}{l}
\mathrm{f} \\
0 \\
0
\end{array}\right\}
$$

where $\mathbf{a}$ and $\mathbf{b}$ are the vectors of modal contributions for the vibration field of leaves $\mathrm{A}$ and $\mathrm{B}$ respectively, $\mathbf{p}$ is the vector of modal contributions for the pressure field in the cavity and $[\mathrm{f}]_{r}=\int_{\Omega_{x y}} q \phi_{r} \mathrm{~d} x \mathrm{~d} y$.

$\mathbf{A}$ and $\mathbf{B}$ are diagonal matrices, whose components are

$$
[\mathrm{A}]_{r r},[\mathrm{~B}]_{r r}=\left(D k_{r}^{4}-\omega^{2} \rho_{s}\right) \int_{\Omega_{x y}} \phi_{r}(x, y) \phi_{r}(x, y) \mathrm{d} x \mathrm{~d} y .
$$

These matrices are associated to the independent behaviour of each leaf.

Due to the orthogonality of the cavity eigenfunctions, $\mathbf{C}$ is also a diagonal matrix, associated to the behaviour of the cavity alone, whose components are

$$
[\mathrm{C}]_{s s}=\left(k^{2}-k_{s}^{2}\right) \int_{\Omega_{c}} \psi_{s}(x, y, z) \psi_{s}(x, y, z) \mathrm{d} \Omega
$$

where

$$
k_{s}^{2}=\left(\frac{s_{x} \pi}{L_{x}}\right)^{2}+\left(\frac{s_{y} \pi}{L_{y}}\right)^{2}+\left(\frac{s_{z} \pi}{H}\right)^{2} .
$$

The matrices that transmit the pressure of the cavity to the leaves are $\mathbf{A}_{\mathbf{C}}$ and $\mathbf{B}_{\mathbf{C}}$, where

$$
\begin{aligned}
{\left[\mathrm{A}_{\mathrm{C}}\right]_{r s} } & =-\int_{\Omega_{x y}} \phi_{r}(x, y) \psi_{s}(x, y, H) \mathrm{d} x \mathrm{~d} y \\
{\left[\mathrm{~B}_{\mathrm{C}}\right]_{r s} } & =\int_{\Omega_{x y}} \phi_{r}(x, y) \psi_{s}(x, y, 0) \mathrm{d} x \mathrm{~d} y .
\end{aligned}
$$

The matrices that transmit the vibration of the leaves to the cavity are $\mathbf{C}_{\mathbf{A}}$ and $\mathbf{C}_{\mathbf{B}}$, where

$$
\begin{aligned}
& {\left[\mathrm{C}_{\mathrm{A}}\right]_{s r}=-\rho \omega^{2} \int_{\Omega_{x y}} \psi_{s}(x, y, H) \phi_{r}(x, y) \mathrm{d} x \mathrm{~d} y} \\
& {\left[\mathrm{C}_{\mathrm{B}}\right]_{s r}=\rho \omega^{2} \int_{\Omega_{x y}} \psi_{s}(x, y, 0) \phi_{r}(x, y) \mathrm{d} x \mathrm{~d} y .}
\end{aligned}
$$

This approach takes into account the boundary conditions of the leaves (4) and the cavity contour (10), but has a disadvantage: the eigenfunctions of the cavity have null normal derivative at the boundaries. Therefore, the continuity of the normal velocity at the fluid-structure interface can only be enforced weakly. 


\section{The finite layer method}

The FLM is presented in this paper as a technique with less computational cost than the FEM but still detailed enough to enforce the interface conditions between fluid and structure. Thus, it is specially suitable for solving the noise transmission through layered configurations of finite dimensions.

The finite layer method is used as a discretisation technique for the pressure field when solving the vibroacoustic problem in the double wall. For the structural part of the problem, the same modal formulation described in Section 2.3 is used.

The weak form of the acoustic problem is

$$
\begin{aligned}
\int_{\Omega_{c}} \nabla p \cdot \nabla v \mathrm{~d} \Omega-k^{2} \int_{\Omega_{c}} p v \mathrm{~d} \Omega & -\int_{\Omega_{x y}} \rho \omega^{2} v(x, y, H)\left(\mathbf{u}_{\mathbf{A}} \cdot \mathbf{n}\right) \mathrm{d} x \mathrm{~d} y- \\
& -\int_{\Omega_{x y}} \rho \omega^{2} v(x, y, H)\left(\mathbf{u}_{\mathbf{B}} \cdot \mathbf{n}\right) \mathrm{d} x \mathrm{~d} y=0 \quad \forall v .
\end{aligned}
$$

The pressure field is interpolated by means of layer functions. These can be understood as standard FEM interpolation functions [46] in the $z$ direction $N_{j}(z)$, multiplied by appropriate interpolation functions $\Phi_{s}(x, y)$ in the $x y$ plane

$$
p(\mathbf{x})=\sum_{s=1}^{n_{x y}} \sum_{j=1}^{n_{z}} p_{j s} N_{j}(z) \Phi_{s}(x, y) .
$$

In Eq. (26), $n_{z}$ is the number of nodes in the $z$ direction as shown in Fig. $4, n_{x y}$ is the number of interpolation functions considered in the $x y$ plane and $p_{j s}$ is the pressure phasor value at node $j$ for the interpolation function $\Phi_{s}(x, y)$. In this work, $\Phi_{s}(x, y)$ is chosen such as to provide the same description in the $x y$ plane as in Section 2.3

$$
\Phi_{s}(x, y)=\cos \left(\frac{s_{x} \pi x}{L_{x}}\right) \cos \left(\frac{s_{y} \pi y}{L_{y}}\right) \quad s_{x}, s_{y}=0,1,2, \ldots
$$

Therefore, the set of functions $\Phi_{s}(x, y)$ satisfies orthogonality and also the condition (10) at the cavity contour.

The test functions $v$ are chosen of the same type as the interpolation functions of Eq. (26)

$$
v(\mathbf{x})=\sum_{t=1}^{n_{x y}} \sum_{i=1}^{n_{z}} v_{i t} N_{i}(z) \Phi_{t}(x, y) .
$$

Eq. (25) must be satisfied for any set of values $v_{i t}$.

With these functions, the operations in Eq. (25) can be split among those in the $x y$ cross-section and those in the $z$ direction. The $x y$ cross-section is treated analytically in order to reduce the problem to a one-dimensional FEM type calculation in the $z$ direction. Thus, $n_{x y}$ sets of $n_{z}$ linear equations are obtained.

As an example, the first term of the weak form (25) and the test function $v=$ $N_{i}(z) \Phi_{t}(x, y)$ are considered. The contribution to the $\{i, j\}$ matrix coefficient corre- 


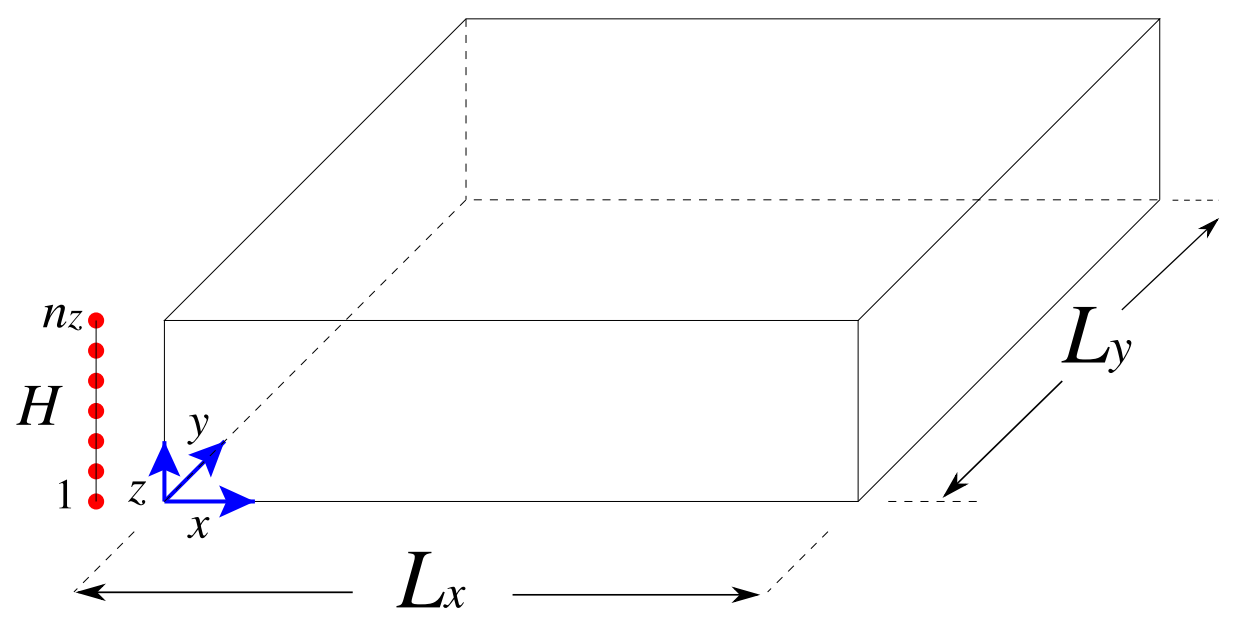

Figure 4: Sketch and notation used in the finite layer method.

sponding to the layer functions $t$ and $s$ can be written as

$$
\begin{aligned}
{\left[\int_{\Omega_{c}} \nabla p \cdot \nabla v \mathrm{~d} \Omega\right]_{t s, i j} } & =\int_{0}^{H} N_{i}^{\prime} N_{j}^{\prime} \mathrm{d} z \int_{\Omega_{x y}} \Phi_{t} \Phi_{s} \mathrm{~d} x \mathrm{~d} y+ \\
+ & \int_{0}^{H} N_{i} N_{j} \mathrm{~d} z \int_{\Omega_{x y}} \nabla_{x y} \Phi_{t} \cdot \nabla_{x y} \Phi_{s} \mathrm{~d} x \mathrm{~d} y \\
& i, j=1,2, \ldots, n_{z} \quad t, s=1,2, \ldots, n_{x y}
\end{aligned}
$$

where $N_{i}^{\prime}=\frac{\mathrm{d} N_{i}}{\mathrm{~d} z}$ and $\nabla_{x y}=(\partial / \partial x, \partial / \partial y)^{T}$ is the gradient in the $x y$ plane. A similar substitution can be done for the other terms of Eq. (25).

Combining the discretised weak form for the cavity pressure with those of the leaf equations, the resulting linear system has the same structure as that of Eq. (17):

$$
\left(\begin{array}{ccc}
\mathrm{A} & \mathrm{A}_{\mathrm{C}} & 0 \\
\mathrm{C}_{\mathrm{A}} & \mathrm{C} & \mathrm{C}_{\mathrm{B}} \\
0 & \mathrm{~B}_{\mathrm{C}} & \mathrm{B}
\end{array}\right)\left\{\begin{array}{l}
\mathrm{a} \\
\mathrm{p} \\
\mathrm{b}
\end{array}\right\}=\left\{\begin{array}{l}
\mathrm{f} \\
0 \\
0
\end{array}\right\}
$$

where $\mathbf{p}$ is the vector with the contributions $p_{j s}$ and $\mathbf{a}, \mathbf{b}$ and $\mathbf{f}$ have already been defined in Section 2.3.

$\mathbf{A}$ and $\mathbf{B}$ are the same matrices of Eq. (18) and $\mathbf{C}$ is the matrix of the cavity alone, which in this case is a block-diagonal matrix. Each block $\{s, s\}$ is

$$
\mathbf{C}_{s s}=\mathbf{K} I_{1}(s, s)+\mathbf{M} I_{2}(s, s)-k^{2} \mathbf{M} I_{1}(s, s),
$$

has $n_{z} \times n_{z}$ size and a tridiagonal structure. The number of non-zero blocks in the matrix is $n_{x y}$. The terms of Eq. (31) are defined in C.

The coupling matrices that transmit the pressure of the cavity to the leaves are $\mathbf{A}_{\mathbf{C}}$ and $\mathbf{B}_{\mathbf{C}}$. They are also block matrices and each block has $1 \times n_{z}$ size. The $j$ component of block $\{r, s\}$ is $\left[\mathrm{A}_{\mathrm{C} r s}\right]_{j}=-N_{j}(H) I_{3}(r, s)$ and $\left[\mathrm{B}_{\mathrm{C} r s}\right]_{j}=N_{j}(0) I_{3}(r, s)$, where $I_{3}(r, s)=\int_{\Omega_{x y}} \phi_{r} \Phi_{s} \mathrm{~d} x \mathrm{~d} y$.

The coupling matrices that transmit the vibration of the leaves to the cavity are $\mathbf{C}_{\mathbf{A}}$ and $\mathbf{C}_{\mathbf{B}}$. They are also block matrices and in this case the size of the blocks 
is $n_{z} \times 1$. The $j$ component of block $\{s, r\}$ is $\left[\mathrm{C}_{\mathrm{A} s r}\right]_{j}=-\rho \omega^{2} N_{j}(H) I_{3}(s, r)$ and $\left[\mathrm{C}_{\mathrm{B} s r}\right]_{j}=\rho \omega^{2} N_{j}(0) I_{3}(s, r)$.

\subsection{Modelling of multilayered double walls}

The FLM can be applied for dealing with multilayered double walls. A particular case of this is a double wall partially filled with absorbing material (with a filling ratio $\beta$ ) as depicted in Fig. 5.

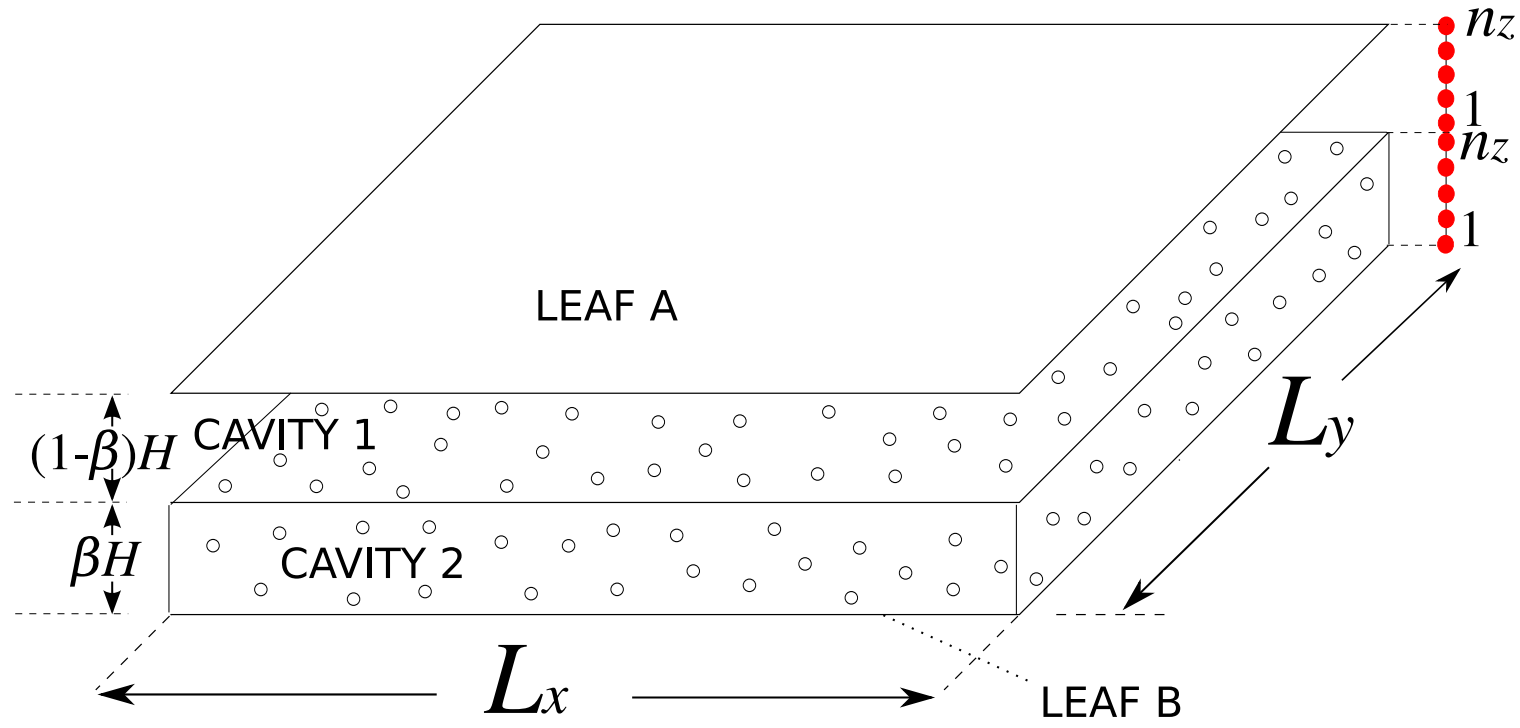

Figure 5: Sketch of the multilayer double wall.

The absorbing material is modelled in this work with the equivalent fluid model suggested by Delany and Bazley [18] and improved by Miki [31]: it is considered as a fluid with complex and frequency-dependent wave number and density. This model is specially suitable for fibrous materials with porosity near 1. For dealing with other types of absorbing materials, more complex models such as those described by Allard and Atalla [6] should be used.

The two fluid phases (air cavity and absorbing material) can be modelled with the finite layer method just by defining two different fluid media (cavity 1 and cavity 2) with the appropriate interface conditions: balance of forces (continuity of the pressure)

$$
\begin{aligned}
& \int_{\Omega_{x y}}\left[p_{1}(x, y, \beta H)-p_{2}(x, y, \beta H)\right] v_{1} \mathrm{~d} \Omega=0 \\
& \int_{\Omega_{x y}}\left[p_{1}(x, y, \beta H)-p_{2}(x, y, \beta H)\right] v_{2} \mathrm{~d} \Omega=0
\end{aligned}
$$

and permanent contact between phases (continuity of the normal velocity)

$$
\begin{gathered}
\mathrm{v}_{n, 1}=-\mathrm{v}_{n, 2} \\
\downarrow \\
\downarrow-\frac{1}{\mathrm{i} \omega \rho_{1}} \nabla_{n} p_{1}(z=\beta H)=\frac{1}{\mathrm{i} \omega \rho_{2}} \nabla_{n} p_{2}(z=\beta H) .
\end{gathered}
$$

In Eq. (33), $\rho_{1}$ and $\rho_{2}$ are the densities of the air and absorbing material respectively (see Fig. 5). In that sense, FLM shows an advantage compared to the modal bases 
of Section 2.3, since the bases of functions allow the enforcement of the interface conditions.

Combining these conditions with the discretised weak forms of the leaf and cavity equations (one for each leaf and one for each cavity), the linear system to solve if the only external excitation is applied to leaf $\mathrm{A}$ is

$$
\left(\begin{array}{cccc}
\mathrm{A} & \mathrm{A}_{\mathrm{C}} & 0 & 0 \\
\mathrm{C}_{\mathrm{A}} & \mathrm{C}_{11} & \mathrm{C}_{12} & 0 \\
0 & \mathrm{C}_{21} & \mathrm{C}_{22} & \mathrm{C}_{\mathrm{B}} \\
0 & 0 & \mathrm{~B}_{\mathrm{C}} & \mathrm{B}
\end{array}\right)\left\{\begin{array}{c}
\mathrm{a} \\
\mathrm{p}_{1} \\
\mathrm{p}_{2} \\
\mathrm{~b}
\end{array}\right\}=\left\{\begin{array}{l}
\mathrm{f} \\
0 \\
0 \\
0
\end{array}\right\}
$$

Matrices $\mathbf{C}_{\mathbf{1 1}}$ and $\mathbf{C}_{\mathbf{2} 2}$ have the structure of matrix $\mathbf{C}$ of Eq. (19) plus some extra terms due to the interface conditions (32) and (33). Matrices $\mathbf{C}_{\mathbf{1 2}}$ and $\mathbf{C}_{\mathbf{2 1}}$ are sparse and carry the rest of the information of Equations (32) and (33). See D for more details on Eq. (34).

\subsection{Truncating the trigonometric series}

A key issue in the modal expansions is to decide which and how many functions are taken into account (see the paper of Gagliardini et al. [23]). This question also arises regarding the trigonometric functions used in finite layer methods.

In these techniques, the trigonometric series must be truncated at some point. For simple problems, such as the vibration analysis of a single plate, the modes located in a range around the frequency of the excitation are enough. However, for the case of a double wall other considerations must be done. Since the wave speed is different in the leaves and in the cavity, when considering the pressure field in the cavity, selected modes are:

- Resonant modes of the cavity: those whose eigenfrequency is around the frequency of the excitation.

- Geometrically coincident modes or critical frequency modes of the cavity: those with a wavelength in the $x y$ plane similar to the resonant vibration wavelengths caused in the leaf by the external excitation. These modes are required in order to reproduce the transmission of sound caused at the critical frequency (joint acceptance).

For instance, when exciting at a certain frequency $f_{0}$, the modes considered in the cavity will be those around $f_{0}$ and those around $f_{i}^{*}$, where $f_{i}^{*}$ are such that $\lambda_{\text {leaf }}=$ $c_{\text {leaf }} / f_{0}=c / f_{i}^{*}=\lambda_{\text {cavity }}^{x y}$. As an example, in Fig. 6 the modal contributions for the cavity at $f_{0}=3000 \mathrm{~Hz}$ are shown. The figure reflects that in this case $f^{*}=1469 \mathrm{~Hz}$.

The same discussion applies when choosing the modes considered for the vibration field in the leaves.

\subsection{Definition of outputs}

All the results in this work are expressed as magnitudes defined in regulations. There are differences between the airborne sound and the impact noise in terms of the system 


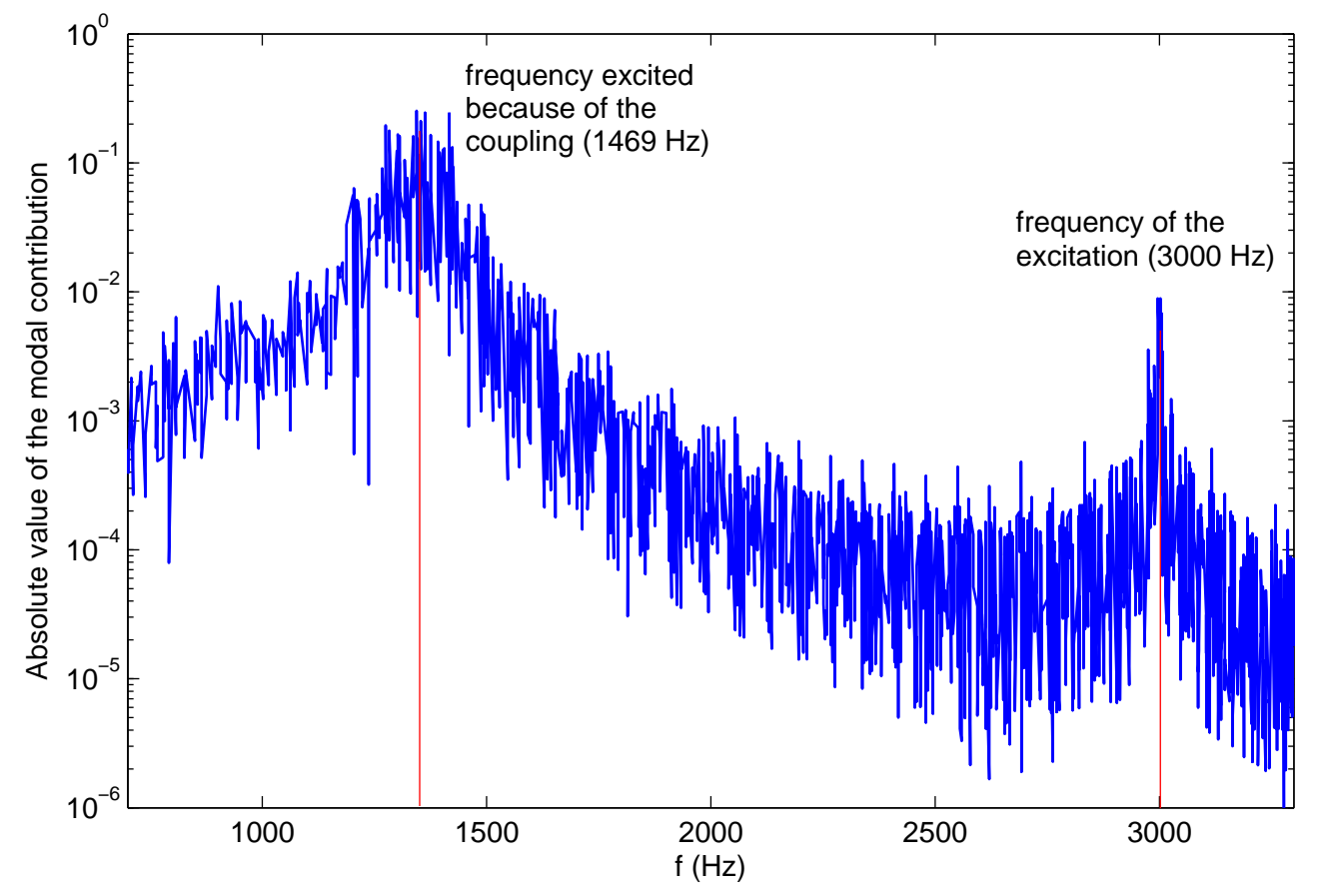

Figure 6: Modal contributions for the cavity at $\mathrm{f}=3000 \mathrm{~Hz}$.

excitation and the required outputs. They are described in Sections 3.3.1 and 3.3.2 respectively.

\subsubsection{Impact noise pressure level}

The impact noise is measured with the normalised impact noise pressure level $L_{\mathrm{n}}$ averaged in one-third octave bands, as defined in ISO 140-6 [3]. For obtaining this value, the excitation must be exerted by the normalised tapping machine [3]. In this work, it is modelled with the expressions provided by Brunskog and Hammer [13]. They suggest that the behaviour of the machine depends on the properties of the contact surface. There are two limit situations in this behaviour. One of them is the case of the hammers rebounding with the same velocity of the impact (elastic behaviour). In the other limit situation the hammers do not rebound at all (damped behaviour). The formulation takes into account the fact that the floor may have an intermediate behaviour between these two limits. It provides the spectrum of the force exerted by the tapping machine $F_{0}(f)$ for a floor of known properties, with

$$
F_{0}(f)=\sum_{n=-\infty}^{\infty} F_{n} \delta\left(f-n f_{r}\right)
$$

with $F_{n}=F_{1}\left(n f_{r}\right) f_{r}, f_{r}=10 \mathrm{~Hz}$ and

$$
F_{1}= \begin{cases}\frac{v_{0} K M}{K-\omega^{2} M+\mathrm{i} \omega K M / R_{r}} & \text { for } K M \geq 4 R_{r}^{2} \\ \frac{v_{0} K M\left(1+e^{-t_{\mathrm{cut}}\left(\mathrm{i} \omega+K / 2 R_{r}\right)}\right)}{K-\omega^{2} M+\mathrm{i} \omega K M / R_{r}} & \text { for } K M<4 R_{r}^{2}\end{cases}
$$


where:

- $v_{0}$ is the speed with which the hammer hits the plate and its value is $v_{0}=$ $\left(2 g h_{0}\right)^{1 / 2}=0.866 \mathrm{~m} \mathrm{~s}^{-1}$ as the hammer is dropped from a height of $0.04 \mathrm{~m}$.

- $K=E D_{h} /\left(1-\nu^{2}\right)$ is the stiffness of the local deformation.

- $D_{h}$ and $M$ are the diameter and the mass of each hammer respectively.

- $R_{r}=8 \sqrt{\rho_{s} B}$ is the input impedance of an infinite plate

- $t_{\text {cut }}=\pi \sqrt{\frac{M}{K}}$ is the time of zero-crossing.

The force is located in four different positions and the resulting radiated power, averaged in each case before computing the impact noise pressure level. With the $(0$ , 0) located at a corner of the plate, these positions are: $\left(0.57 L_{x}, 0.57 L_{y}\right),(0.19$ $\left.L_{x}, 0.19 L_{y}\right),\left(0.19 L_{x}, 0.57 L_{y}\right),\left(0.35 L_{x}, 0.35 L_{y}\right)$.

The noise level is computed in terms of the power radiated by the unexcited leaf as Brunskog and Hammer do in [14]

$$
L_{\mathrm{n}}=10 \log _{10}\left(\frac{\Pi_{\mathrm{rad}}}{p_{\mathrm{ref}}^{2}} \frac{4 \rho c}{A_{0}}\right) \mathrm{dB}
$$

where $A_{0}$ is the reference absorption area $\left(10 \mathrm{~m}^{2}\right.$ for dwellings $), \Pi_{\text {rad }}$ the radiated power and $p_{\text {ref }}$ the reference pressure $\left(2 \times 10^{-5} \mathrm{~Pa}\right)$.

As proposed by Williams [44], the power radiated by the unexcited leaf is expressed in terms of the leaf surface velocity as

$$
\Pi_{\mathrm{rad}}=\frac{\omega \rho}{4 \pi} \int_{\Omega_{x y}^{\prime}} \int_{\Omega_{x y}} \hat{v}\left(x^{\prime}, y^{\prime}\right) \hat{v}^{*}(x, y) \frac{\sin (k r)}{r} \mathrm{~d} x \mathrm{~d} y \mathrm{~d} x^{\prime} \mathrm{d} y^{\prime}
$$

where $\hat{v}$ is the velocity field, $r=\left[\left(x-x^{\prime}\right)^{2}+\left(y-y^{\prime}\right)^{2}\right]^{1 / 2}$, the symbol * means the complex conjugate and $\Omega_{x y}, \Omega_{x y}^{\prime}$ are used to denote the double integral over the leaf.

This integral is calculated numerically with the trapezoidal composite rule, using six nodes per wavelength. However, at large frequencies ( $f$ greater than $708 \mathrm{~Hz}$ in the examples of Section 4.2), the computation of the power is simplified by means of the expressions shown in [35]. In them, the radiation efficiency $\sigma_{\text {rad }}$ depends on the excitation frequency, the properties of the structure and the medium into which sound is radiated. With these expressions, the radiated power is computed as

$$
\Pi_{\mathrm{rad}}=R_{\mathrm{rad}}\left\langle v_{R M S}^{2}\right\rangle
$$

where $\left\langle v_{R M S}^{2}\right\rangle$ is the spatial mean square value of the leaf vibration velocity and $R_{\text {rad }}=\sigma_{\text {rad }} \rho c S$ is the radiation resistance (with $S$ the surface of the leaf). This combination of techniques was used in [19] and reduces significantly the computational cost of the calculation. 


\subsubsection{Sound reduction index}

The airborne sound is measured with the sound reduction index $R$. Models of Section 2.1 provide directly the transmission loss and, with Eq. (2), $R$ can be obtained. In the rest of the approaches, this value is computed in terms of the incident and radiated powers, $\Pi_{\text {in }}$ and $\Pi_{\mathrm{rad}}$, of the structure.

The computation of this value requires a pressure wave impinging on one of the leaves, modelled as

$$
p(\mathbf{x})=p_{0} \mathrm{e}^{-\mathrm{i}\left(k_{x} x+k_{y} y+k_{z} z\right)}
$$

where $k_{x}=k \sin \varphi \cos \theta, k_{y}=k \sin \varphi \sin \theta$ and $k_{z}=k \cos \varphi$.

This wave may have several orientations, defined by angles $\theta$ and $\varphi$ as shown in Fig. 7. Four different values of $\theta$, equispaced between $\theta=0$ and $\theta=45^{\circ}$ due to the symmetry of the problem, are considered. If the leaf was rectangular instead of square, this limit would be $90^{\circ}$. Also ten different values of $\varphi$ have been considered, equispaced between $\varphi=0$ and $\varphi_{\lim }=90^{\circ}$ for the finite size models, and $\varphi_{\lim }=78^{\circ}$ for the two impedance models, in order to reproduce a diffuse incident field.

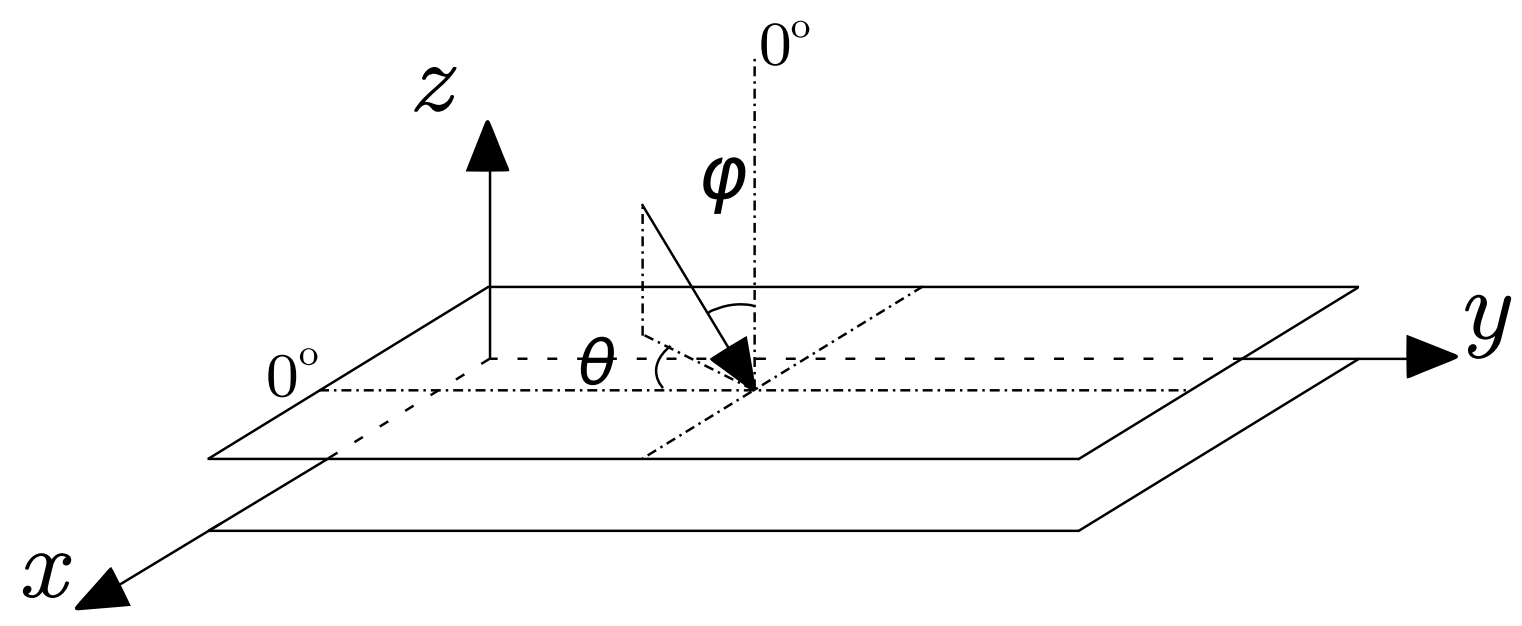

Figure 7: Incident angles.

The final value of the sound reduction index is computed as

$$
R=10 \log _{10}\left(\frac{1}{\tau_{\text {diff }}}\right)
$$

where

$$
\tau_{\text {diff }}=\frac{\int_{0}^{\theta=45^{\circ}} \int_{0}^{\varphi_{\mathrm{lim}}} \tau(\theta, \varphi) \cos (\theta) \sin (\theta) \cos (\varphi) \sin (\varphi) \mathrm{d} \varphi \mathrm{d} \theta}{\int_{0}^{\theta=45^{\circ}} \int_{0}^{\varphi_{\lim }} \cos (\theta) \sin (\theta) \cos (\varphi) \sin (\varphi) \mathrm{d} \varphi \mathrm{d} \theta}
$$

and

$$
\tau(\theta, \varphi)=\frac{\Pi_{\mathrm{rad}}(\theta, \varphi)}{\Pi_{\mathrm{in}}(\theta, \varphi)} .
$$

In Eq. (43), $\Pi_{\mathrm{rad}}(\theta, \varphi)$ is obtained with the same technique described in Section 3.3.1 and

$$
\Pi_{\text {in }}(\theta, \varphi)=\frac{\left\langle P_{\mathrm{RMS}}^{2}\right\rangle L_{x} L_{y} \cos \varphi}{\rho c},
$$

where $\left\langle P_{\mathrm{RMS}}^{2}\right\rangle$ is the mean square pressure exciting the leaf. 


\section{Simulations and comparisons}

In this section, the FLM is compared both with experimental data and with the other models described in Section 2. An example of the possible applications of the finite layer method is also shown: a double wall with the cavity partially filled with absorbent material.

\subsection{Comparison with experimental data}

The use of the FLM for modelling the sound transmission in double walls is tested by comparing it with available experimental data. In [38], Tadeu et al. show the sound reduction index measured in the lab for a double glazing. In Table 1 the properties of the glass leaves are shown. The cavity between them is $0.012 \mathrm{~m}$ thick.

\begin{tabular}{lll}
\hline Variable & Symbol & Value \\
\hline Leaf size, $x$ direction & $L_{x}$ & $1.2 \mathrm{~m}$ \\
Leaf size, $y$ direction & $L_{y}$ & $1.2 \mathrm{~m}$ \\
Thickness & $h$ & $0.004 \mathrm{~m}$ \\
Young's modulus & $E_{\text {leaf }}$ & $7.2 \times 10^{10} \mathrm{~N} \mathrm{~m}^{-2}$ \\
Density & $\rho_{\text {leaf }}$ & $2500 \mathrm{~kg} \mathrm{~m}^{-3}$ \\
Poisson's ratio & $\nu$ & 0.22 \\
Loss factor & $\eta$ & $4 \%$ \\
\hline
\end{tabular}

Table 1: Properties of the double glazing.

The experimental results in [38] are depicted averaged in 1/10 octave bands. For the comparison, their sound energies have been averaged in order to provide the sound reduction index law in one-third octave bands

$$
\langle R\rangle=10 \log _{10}\left[\frac{1}{n} \sum_{i=1}^{n} 10^{0.1 R_{i}}\right] .
$$

In Fig. 8 the comparison between the simulation with FLM and the experimental results is shown. The good performance of the numerical method is verified, since the trend of the numerical results coincides with that of the experimental values.

\subsection{Comparison with other models}

All the methods described in Section 2 are compared here with the finite layer method. Both the impact noise and the airborne sound are calculated for a wood double wall with an empty cavity.

Table 2 shows the main features of the five techniques compared in this paper, according to different criteria. Among them, are the reflecting boundary conditions (10) at the cavity contour and the use of the Helmholtz equation for modelling the pressure field in the cavity.

For all the comparisons, the properties of the leaves are defined in Table 3. The cavity is $0.07 \mathrm{~m}$ thick. 


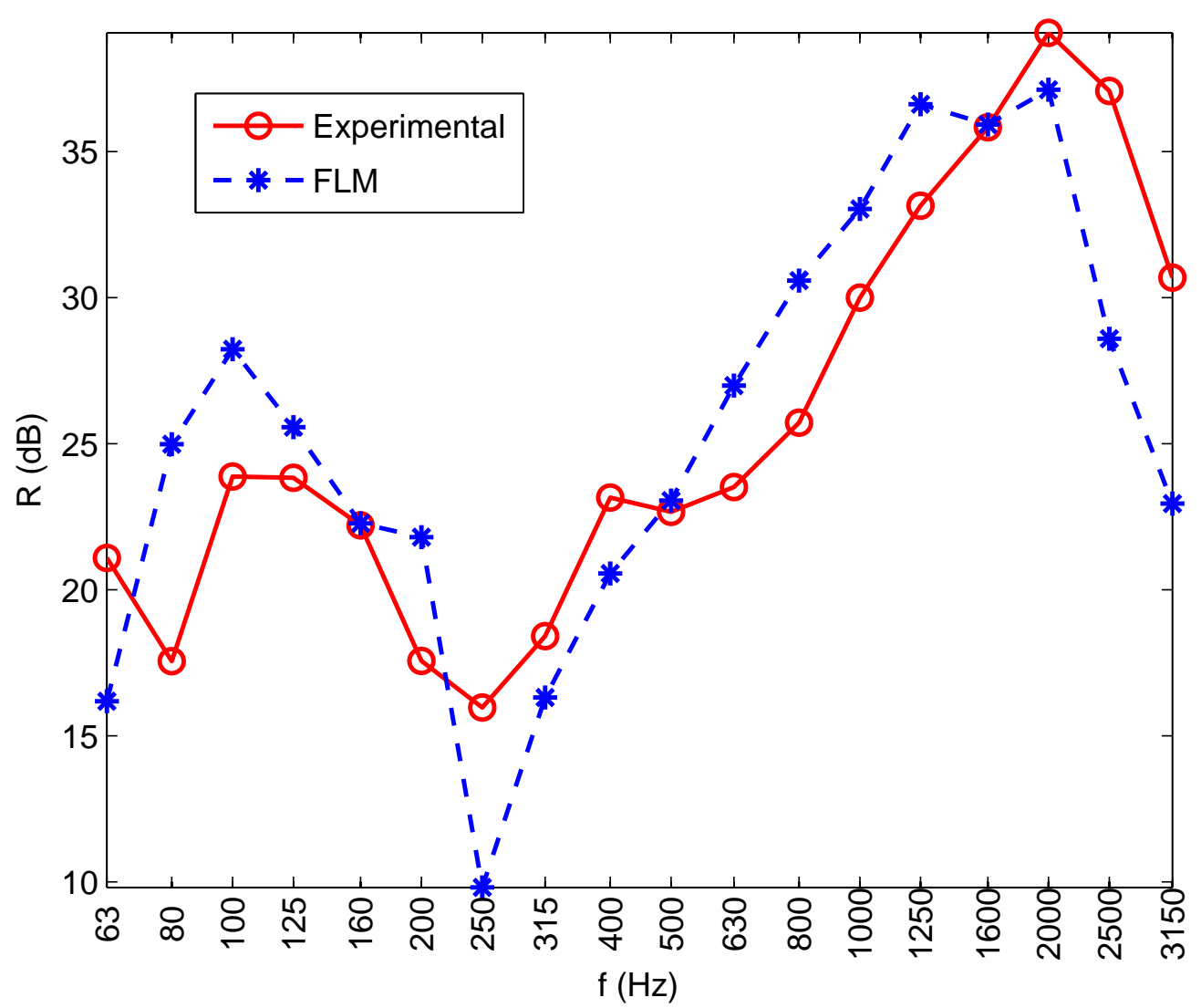

Figure 8: Comparison with experimental measurements.

\subsubsection{Comparison for the impact noise}

First, the impact noise associated to the wood double wall is computed and shown in Fig. 9. In this case only the models presented in Sections 2.2 and 2.3 are compared with the finite layer method. The simple impedance models of Section 2.1 are not considered because they only provide the transmission loss and, therefore, only model the transmission of airborne sound.

In Fig. 9, the results provided by the FLM and the discretisation with modal bases are essentially the same. However, the adaptation of the model of Xin et al. provides slightly different results.

The main conclusion to be drawn here is that using interpolation functions with zero derivative at the fluid-structure interface for the air cavity (modal bases) is not a problem for this example. Not imposing strongly the continuity of the normal velocity does not affect the result.

Fig. 9 also shows that the description of the pressure field of Xin et al. provides a slightly different impact noise law. In particular, at the band of $125 \mathrm{~Hz}$, the effect of the mass-spring-mass resonance is more pronounced due to the use of the same in-plane functions in leaves and cavity. The conclusion here is that the boundary conditions at the cavity contour are the main responsibles of the different performance of the model of Xin et al. The influence of the boundary conditions at the cavity 


\begin{tabular}{lccccc}
\hline Hypothesis & Fahy & $\begin{array}{c}\text { Au and } \\
\text { Byrne }\end{array}$ & $\begin{array}{c}\text { Adapt. } \\
\text { of Xin } \\
\text { et } \boldsymbol{a l} .\end{array}$ & $\begin{array}{c}\text { Modal } \\
\text { bases }\end{array}$ & FLM \\
\hline $\begin{array}{l}\text { Finite size of } \\
\text { the leaves }\end{array}$ & No & No & Yes & Yes & Yes \\
\hline $\begin{array}{l}\text { Continuity of normal } \\
\text { velocity at interfaces }\end{array}$ & No & No & Yes & No & Yes \\
\hline $\begin{array}{l}\text { Reflecting boundary } \\
\text { conditions for the cavity }\end{array}$ & No & No & No & Yes & Yes \\
\hline $\begin{array}{l}\text { Thin plate equation } \\
\text { for the leaves }\end{array}$ & No & No & Yes & Yes & Yes \\
\hline $\begin{array}{l}\text { Helmholtz equation } \\
\text { for the cavity }\end{array}$ & No & No & No & Yes & Yes \\
\hline $\begin{array}{l}\text { Dimensionality of } \\
\text { the pressure field }\end{array}$ & 1D & 1D & $3 \mathrm{D}$ & $3 \mathrm{D}$ & $3 \mathrm{D}$ \\
\hline
\end{tabular}

Table 2: Hypotheses of each technique

\begin{tabular}{lll}
\hline Variable & Symbol & Value \\
\hline Leaf size, $x$ direction & $L_{x}$ & $2.4 \mathrm{~m}$ \\
Leaf size, $y$ direction & $L_{y}$ & $2.4 \mathrm{~m}$ \\
Thickness & $h$ & $0.02 \mathrm{~m}$ \\
Young's modulus & $E_{\text {leaf }}$ & $10^{10} \mathrm{~N} \mathrm{~m}^{-2}$ \\
Density & $\rho_{\text {leaf }}$ & $400 \mathrm{~kg} \mathrm{~m}^{-3}$ \\
Poisson's ratio & $\nu$ & 0.25 \\
Loss factor & $\eta$ & $5 \%$ \\
\hline
\end{tabular}

Table 3: The assumed properties for a wood leaf, used for all the analysis.

contour is larger than that of the interface conditions at the fluid-structure contact.

A remark must be done here in terms of the computational cost. The number of degrees of freedom inside the air cavity for the analysis with modal bases at $3400 \mathrm{~Hz}$ (one of the highest frequencies considered) is 4340. If the discretisation is done with FLM the system has 22934 degrees of freedom. This value is about six times larger than that of the modal analysis. This is due to the wave behaviour of the sound: for that cavity size, at a frequency of $3400 \mathrm{~Hz}$, the wavelength of the pressure field is of the same size of the thickness of the cavity. Hence, it is reproducible with only one trigonometric function in the $z$ direction. However, for the same wave, six nodes are required in that direction in the FEM-like approach of FLM (six times more degrees of freedom). Finally, if the computation was done with pure finite elements along the cavity, respecting the rule of six elements per wavelength, the required number of degrees of freedom would be around 200000 . The quantitative reduction due to the use of trigonometric functions is significant.

The optimal approach for the acoustic behaviour of double walls with empty cav- 


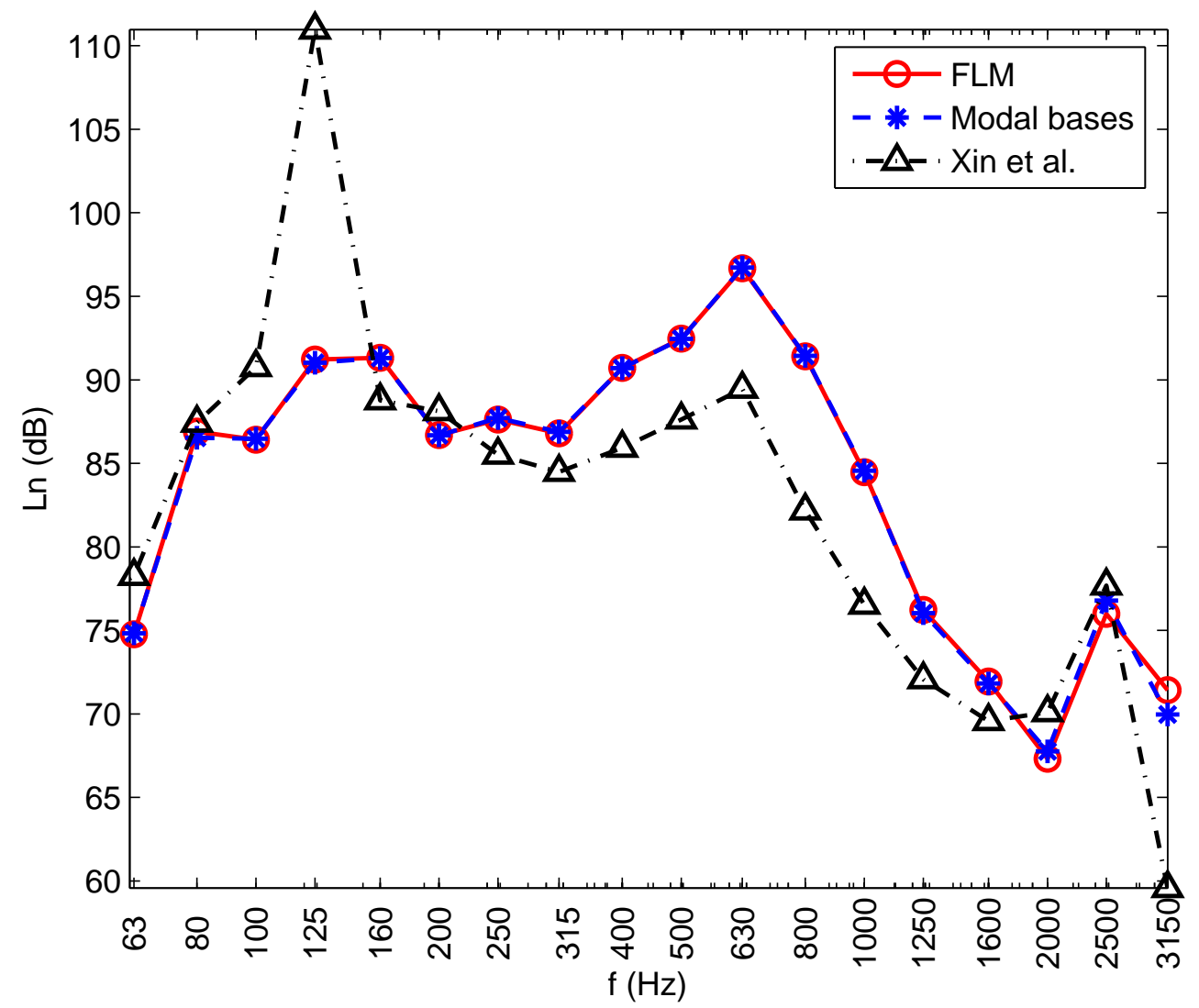

Figure 9: Comparisons of models for the impact noise of the double wall.

ities would be the analysis with modal bases.

\subsubsection{Comparison for the airborne sound}

In Fig. 10 the sound reduction index provided by the wood double wall is shown, computed with all the models described in this work and averaged in one-third octave bands.

For frequencies larger than the coincidence frequency of the leaves $\left(f_{c}=616.5\right.$ $\mathrm{Hz}$ ), the two discretisation-driven techniques, the adaptation of the method of Xin et al. and the impedance model of $\mathrm{Au}$ and Byrne provide the same trend in the results. The impedance model provided by Fahy captures the shape of the sound reduction index law but not the values provided by the other models. For frequencies lower than $f_{c}$, the discretisation-driven techniques provide different values than the others. This shows that the assumptions of the other methods, shown in Table 2, are not satisfied at low frequencies. In particular, modelling the fluid with the Helmholtz equation and respecting the size and boundary conditions of the wall is more important than enforcing the continuity of the normal velocity at the fluid-structure interface. 


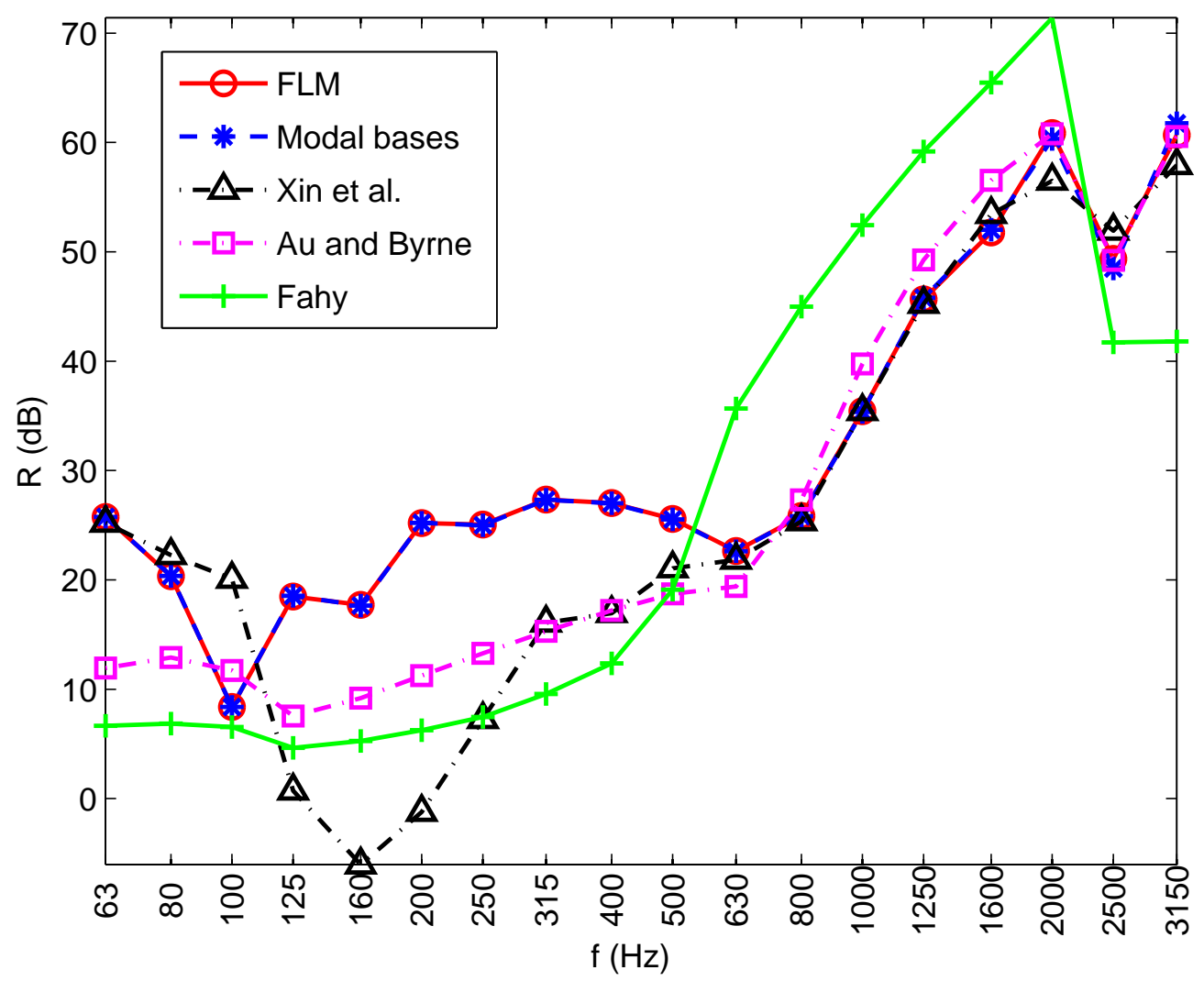

Figure 10: Comparisons of models for the sound reduction index of the double wall.

\subsection{Modelling of multilayered double walls}

The sound reduction index caused by a double wall with different filling ratios of absorbing material is computed. The properties of the double wall are the same as those of Section 4.2. The resistivity of the absorbing material is $\sigma=10000 \mathrm{~N} \mathrm{~s} \mathrm{~m}^{-4}$, the distance between the two leaves is $0.07 \mathrm{~m}$ and different values of $\beta$ are simulated: $0,0.25,0.5$ and 1 . Since the equivalent fluid model is not recommended for $f<0.01 \sigma$, in Fig. 11 the sound reduction index is only shown for frequencies larger than $100 \mathrm{~Hz}$.

Fig. 11 illustrates the insulating effect of the absorbing material. Higher ratios of absorbing material inside the cavity cause an increase in the sound reduction index, specially at low frequencies. However, between 1000 and $2000 \mathrm{~Hz}$, the variations in the sound reduction index caused by the absorbing material are not larger than 10 dB.

The influence of the position of the absorbing layer has also been analysed: the simulations with the upper half of the cavity filled with absorbing material provide the same results as those with the absorbing material in the lower part. The position of the absorbing layer has no influence at all in the sound reduction index. The FLM can be used in the same way for any filling ratio and material, just by changing the size and properties of the cavities. Thus, it is especially suitable for this type of analyses. 


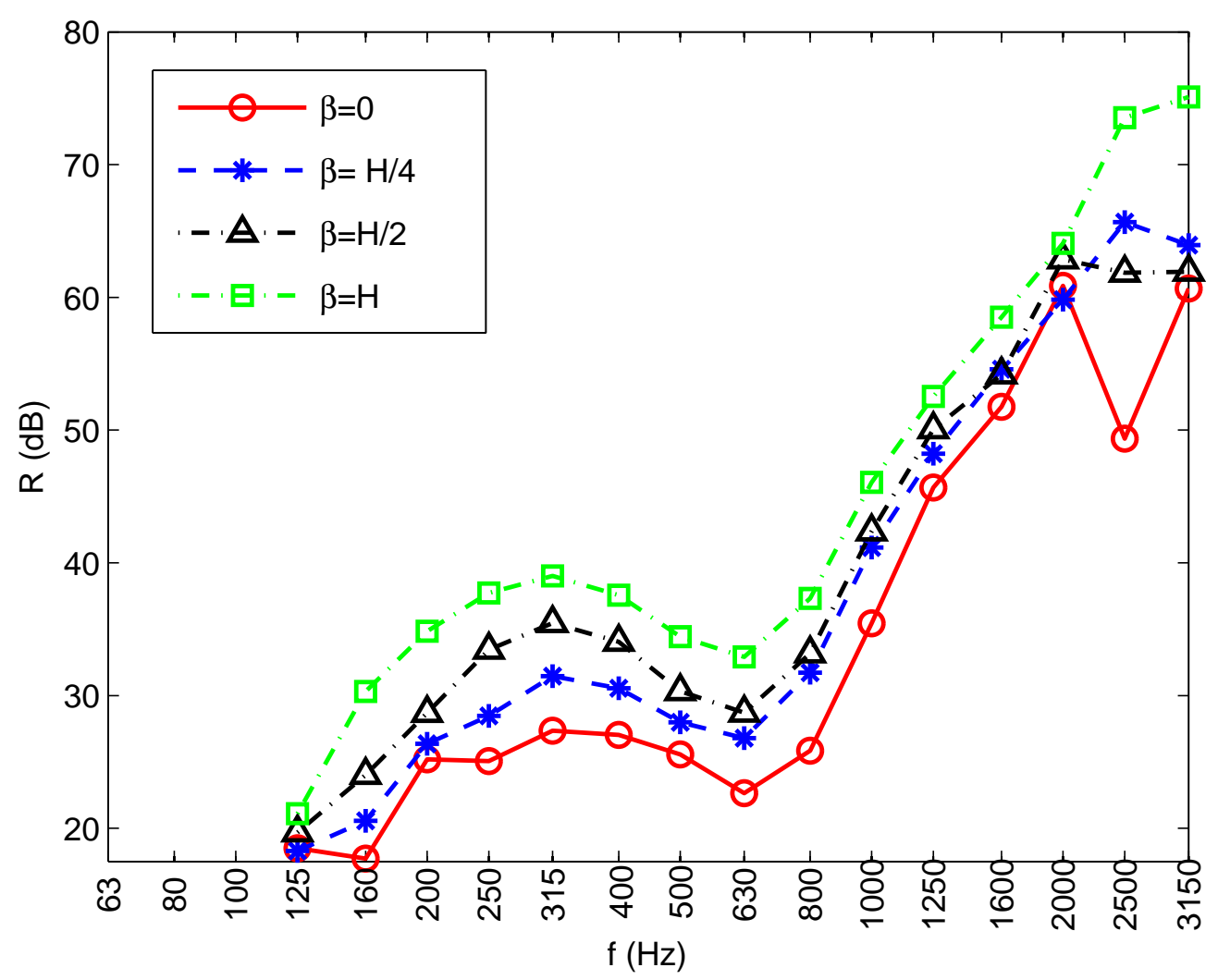

Figure 11: Effect of the absorbing ratio inside the cavity.

\section{Discussion}

The simulations of Section 4 show that the finite layer method is a useful technique for modelling the sound transmission through double walls. It provides good results for different excitations and cavity fillings if modes are chosen properly. Results show a good agreement when comparing them with experimental data.

The results provided by the FLM coincide with those of the modal discretisation for the case of double walls with an air cavity in between. Other techniques, such as the adaptation of the method of Xin et al. or the impedance method of $\mathrm{Au}$ and Byrne, provide good results for frequencies higher than the coincidence frequency but poor results for lower frequencies. Simpler models such as that of Fahy only capture the shape of the law but not the sound reduction level.

The different assumptions of each approach cause differences in the noise transmission predicted by them. The effect of the leaf dimensions in the sound insulation of the wall is relevant for frequencies lower than the coincidence frequency.

The boundary conditions of the wall are also specially important at low frequencies. Therefore, the only methods that provide good results throughout the whole frequency range are those based on the differential equations: thin plate for the leaves and Helmholtz for the cavity.

For air cavities, there is no difference between the results of modal analysis or 
FLM. Thus, the best choice is modal analysis because of its lower computational cost.

The null derivative of the modal interpolation functions at the fluid-structure interface does not affect the results for empty cavities. However, it becomes relevant when dealing with two fluid-like layers. With a cosine series there is no way of enforcing the continuity of the normal velocity at the interface between fluids, not even weakly. The basis of functions should be enriched with functions with a sine in the $z$ direction, such as

$$
\psi_{s}(\mathbf{x})=\cos \left(\frac{s_{x} \pi x}{L_{x}}\right) \cos \left(\frac{s_{y} \pi y}{L_{y}}\right) \sin \left(\frac{s_{z} \pi z}{H}\right)
$$

This enrichment would double the number of unknowns in the cavity and also cause the loss of the basis orthogonality. Hence, matrices $\mathbf{C}_{\mathbf{1 1}}$ and $\mathbf{C}_{\mathbf{2 2}}$ would be full, as well as matrices $\mathbf{C}_{12}$ and $\mathbf{C}_{\mathbf{2 1}}$. The FEM-like functions of FLM in the direction perpendicular to the wall, meanwhile, allow the enforcement of the continuity between any type of layer with very sparse matrices $\mathbf{C}_{\mathbf{1 2}}, \mathbf{C}_{\mathbf{2 1}}, \mathbf{C}_{\mathbf{1 1}}$ and $\mathbf{C}_{\mathbf{2 2}}$.

The models presented here can be enriched in different ways: one option is the addition of stiffening elements to the structure. This enrichment can be done in an straightforward way as shown in [19, 36].

Another option is to account for an absorbing cavity contour. The cosine series only allows the description of pressure fields with a null normal velocity in the cavity contour. The absorbing boundary condition

$$
-\nabla p \cdot \mathbf{n}=\rho A \mathrm{i} \omega p
$$

where $A$ is the absorption coefficient of the wall, would have to be enforced weakly in the same way as the interface conditions in Section 2.3.

\section{Conclusions}

- The finite layer method is a reliable technique to model the sound transmission through double walls, specially when dealing with multilayer walls with different fluid-like materials.

- Accounting for the size and boundary conditions of the wall becomes a relevant aspect when modelling the sound transmission at low frequencies.

- Capturing well the continuity of the normal velocity is not relevant for modelling the sound transmission through double walls with empty air cavity.

\section{A Fahy 1985}

In this model

$$
\tau(\varphi)=\left|\frac{\bar{p}_{\text {trans }}}{\bar{p}_{\text {inc }}}\right|^{2}=\left|-\frac{2 \mathrm{i} Z_{0}^{2} \sin (k H \cos \varphi) / \cos ^{2} \varphi}{\overline{z_{1}^{\prime} z_{2}^{\prime}} \sin ^{2}(k H \cos \varphi)+Z_{0}^{2} / \cos \varphi}\right|^{2}
$$


where $p_{\text {trans }}$ is the pressure of transmitted sound, $p_{\text {inc }}$ is the pressure of the incident sound, $Z_{0}=\rho c$ is the characteristic impedance of the air,

$$
\overline{z_{i}^{\prime}}=\overline{z_{i}}+Z_{0} \frac{1-\mathrm{i} / \tan (k H \cos \varphi)}{\cos \varphi} \quad \text { and } \quad \overline{z_{i}}=\mathrm{i} \omega \rho_{s, i}+\eta_{i} \omega_{0} \rho_{s, i}-\mathrm{i} \frac{\omega_{0, i}^{2}}{\rho_{s, i} \omega} \text {. }
$$

Here $\omega_{0}$ is the first eigenfrequency of the leaves.

\section{B Au and Byrne 1987}

In this model the input impedance of the receiving room is defined as $Z_{I}=Z_{0} / \cos \varphi$. This term is the terminal impedance of the excited leaf. The impedances of leaves $B$ and A (layers 2 and 4 respectively) are:

$$
\begin{aligned}
& Z_{2}=Z_{1}+\eta_{2} D_{2} k_{x}^{4} / \omega+\mathrm{i}\left(\omega \rho_{s, 2}-D_{2} k_{x}^{4} / \omega\right) \\
& Z_{4}=Z_{3}+\eta_{1} D_{1} k_{x}^{4} / \omega+\mathrm{i}\left(\omega \rho_{s, 1}-D_{1} k_{x}^{4} / \omega\right) .
\end{aligned}
$$

The impedance of the cavity is

$$
Z_{3}=Z_{c} \frac{\Gamma_{c}}{\Gamma_{c y}} \frac{\left(1+\frac{Z_{c} \Gamma_{c}}{Z_{2} \Gamma_{c y}}\right) \exp \left(\mathrm{i} \Gamma_{c y} H\right)+\left(1-\frac{Z_{c} \Gamma_{c}}{Z_{2} \Gamma_{c y}}\right) \exp \left(-\mathrm{i} \Gamma_{c y} H\right)}{\left(1+\frac{Z_{c} \Gamma_{c}}{Z_{2} \Gamma_{c y}}\right) \exp \left(\mathrm{i} \Gamma_{c y} H\right)-\left(1-\frac{Z_{c} \Gamma_{c}}{Z_{2} \Gamma_{c y}}\right) \exp \left(-\mathrm{i} \Gamma_{c y} H\right)}
$$

where $\Gamma_{c y}^{2}=\Gamma_{c}^{2}-k_{x}^{2}, k_{x}=k \sin \varphi, Z_{c}=Z_{0} \sqrt{1-\mathrm{i} \sigma / \rho \omega}, \Gamma_{c}=k \sqrt{1-\mathrm{i} \sigma / \rho \omega}, \sigma$ is the resistivity of the cavity absorbent ( $\sigma=0$ for air cavities) and $\eta_{i}$ is the damping of layer $i$.

$p_{4}=p_{i} 2 A /(A+1)$ is the input pressure for layer 3 (cavity), $A=Z_{4} \cos \varphi / Z_{0}$. The transmitted pressure over an impervious leaf is: $p_{2}=p_{1} Z_{2} / Z_{1}$ (leaf B), $p_{4}=p_{3} Z_{4} / Z_{3}$ (leaf $A$ ), and the transmitted pressure over the cavity absorbent is calculated by

$$
p_{2}=\frac{p_{3}}{2}\left[\left(1+\frac{Z_{c} \Gamma_{c}}{Z_{3} \Gamma_{c y}}\right) \exp \left(-\mathrm{i} \Gamma_{c y} H\right)+\left(1-\frac{Z_{c} \Gamma_{c}}{Z_{3} \Gamma_{c y}}\right) \exp \left(\mathrm{i} \Gamma_{c y} H\right)\right]
$$

and the transmission loss is computed as

$$
\begin{aligned}
\tau(\varphi)=\left|\frac{p_{1}}{p_{i}}\right|^{2}=\frac{Z_{1} Z_{3}}{Z_{2} Z_{4}} \frac{A}{A+1}\left[\left(1+\frac{Z_{c} \Gamma_{c}}{Z_{3} \Gamma_{c y}}\right) \exp \left(-\mathrm{i} \Gamma_{c y} H\right)+\right. \\
\left.+\left(1-\frac{Z_{c} \Gamma_{c}}{Z_{3} \Gamma_{c y}}\right) \exp \left(\mathrm{i} \Gamma_{c y} H\right)\right] .
\end{aligned}
$$

\section{Finite layer expressions}

In Eq. (31),

$$
\begin{aligned}
I_{1}(s, s)=\int_{\Omega_{x y}} \Phi_{s} \Phi_{s} \mathrm{~d} x \mathrm{~d} y & = \\
& =\int_{0}^{L_{x}}\left[\cos \left(\frac{s_{x} \pi x}{L_{x}}\right)\right]^{2} \mathrm{~d} x \int_{0}^{L_{y}}\left[\cos \left(\frac{s_{y} \pi y}{L_{y}}\right)\right]^{2} \mathrm{~d} y
\end{aligned}
$$




$$
\begin{aligned}
& I_{2}(s, s)=\int_{\Omega_{x y}} \nabla_{x y} \Phi_{s} \cdot \nabla_{x y} \Phi_{s} \mathrm{~d} x \mathrm{~d} y= \\
&=\int_{0}^{L_{x}}\left[-\frac{s_{x} \pi}{L_{x}} \sin \left(\frac{s_{x} \pi x}{L_{x}}\right)\right]^{2} \mathrm{~d} x \int_{0}^{L_{y}}\left[\cos \left(\frac{s_{y} \pi y}{L_{y}}\right)\right]^{2} \mathrm{~d} y+ \\
& \quad+\int_{0}^{L_{x}}\left[\cos \left(\frac{s_{x} \pi x}{L_{x}}\right)\right]^{2} \mathrm{~d} x \int_{0}^{L_{y}}\left[-\frac{s_{y} \pi}{L_{y}} \sin \left(\frac{s_{y} \pi y}{L_{y}}\right)\right]^{2} \mathrm{~d} y
\end{aligned}
$$

and $\mathbf{M}, \mathbf{K}$ are the one-dimensional mass and stiffness matrices defined as

$$
\begin{aligned}
{[\mathrm{K}]_{i j} } & =\int_{0}^{H} N_{i}^{\prime} N_{j}^{\prime} \mathrm{d} z \\
{[\mathrm{M}]_{i j} } & =\int_{0}^{H} N_{i} N_{j} \mathrm{~d} z .
\end{aligned}
$$

\section{Multilayer formulation}

In system (34), matrices $\mathbf{C}_{\mathbf{1 1}}$ and $\mathbf{C}_{\mathbf{2 2}}$ are associated to the air and absorbing material layers respectively. $\mathbf{C}_{\mathbf{1 1}}$ consists of two types of contributions: the same matrix $\mathbf{C}$ defined in Eq. (31) and a term related to Eq. (32) that is assembled at

$$
\left[\mathrm{C}_{11 s t}\right]_{i j}=\left[\mathrm{C}_{11 s t}\right]_{i j}+N_{i}(\beta H) N_{j}(\beta H) I_{1}(s, t),
$$

where $I_{1}(s, t)=\int_{\Omega_{x y}} \Phi_{s} \Phi_{t} \mathrm{~d} x \mathrm{~d} y$.

Analogously, matrix $\mathbf{C}_{\mathbf{2 2}}$ consists of two contributions: on the one hand, the matrix defined in Eq. (31) adapted with the new value of the speed of sound $c_{2}$ and, on the other hand, a term related to Eq. (32) that is assembled at

$$
\left[\mathrm{C}_{22 s t}\right]_{i j}=\left[\mathrm{C}_{22 s t}\right]_{i j}-N_{i}(\beta H) N_{j}(\beta H) I_{1}(s, t) .
$$

Matrix $\mathbf{C}_{\mathbf{1 2}}$ has the information of the continuity of the normal velocity at the interface between the two layers as

$$
\left[\mathrm{C}_{12 s t}\right]_{i j}=\left.\frac{\rho_{1}}{\rho_{2}} N_{i}(\beta H) \frac{\mathrm{d} N_{j}}{\mathrm{~d} z}\right|_{z=\beta H} I_{1}(s, t),
$$

and also the rest of the information of Eq. (32) for cavity 2

$$
\left[\mathrm{C}_{12 s t}\right]_{i j}=\left[\mathrm{C}_{12 s t}\right]_{i j}-N_{i}(\beta H) N_{j}(\beta H) I_{1}(s, t) .
$$

Matrix $\mathbf{C}_{\mathbf{2 1}}$ has the information of Eq. (33) as

$$
\left[\mathrm{C}_{21 s t}\right]_{i j}=-\left.\frac{\rho_{2}}{\rho_{1}} N_{i}(\beta H) \frac{\mathrm{d} N_{j}}{\mathrm{~d} z}\right|_{z=\beta H} I_{1}(s, t),
$$

and also the rest of the information of Eq. (32) for cavity 2

$$
\left[\mathrm{C}_{21 s t}\right]_{i j}=\left[\mathrm{C}_{21 s t}\right]_{i j}+N_{i}(\beta H) N_{j}(\beta H) I_{1}(s, t) .
$$

Matrices $\mathbf{A}, \mathbf{B}, \mathbf{A}_{\mathbf{C}}, \mathbf{B}_{\mathbf{C}}$ and $\mathbf{C}_{\mathbf{A}}$ are the same as defined in Eq. (30). Matrix $\mathbf{C}_{\mathbf{B}}$ is also the same as in Eq. (30) but with density $\rho_{2}$ instead of $\rho$. 


\section{Acknowledgements}

The financial support of the Ministerio de Educación y Ciencia (FPU scholarship program) and the Col-legi d'Enginyers de Camins, Canals i Ports is gratefully acknowledged.

\section{References}

[1] ISO 717-1:1997, Acoustics - Rating of sound insulation in buildings and of building elements - Part 2: Airborne sound insulation. Geneva, Switzerland, 1997.

[2] ISO 717-2:1997, Acoustics - Rating of sound insulation in buildings and of building elements - Part 2: Impact sound isolation. Geneva, Switzerland, 1997.

[3] ISO 140-6:1998, Acoustics - Measurement of sound insulation in buildings and of building elements - Part 6: Laboratory measurements of impact sound insulation of floors. Geneva, Switzerland, 1998.

[4] B. Aalami. Waves in prismatic guides of arbitrary cross section. Journal of Applied Mechanics, 40(4):1067-1072, 1973.

[5] J. Alba, J. Ramis, and V. J. Sánchez-Morcillo. Improvement of the prediction of transmission loss of double partitions with cavity absorption by minimization techniques. Journal of Sound and Vibration, 273(4-5):793-804, 2004.

[6] J.F. Allard and N. Atalla. Propagation of sound in porous media: modelling sound absorbing materials. Wiley, 2009.

[7] A.C.K. Au and K.P. Byrne. On the insertion losses produced by plane acoustic lagging structures. The Journal of the Acoustical Society of America, 82(4):13251333, 1987.

[8] L.L. Beranek. Noise and vibration control. Institute of Noise Control Engineering, 1988.

[9] L.L. Beranek and G.A. Work. Sound transmission through multiple structures containing flexible blankets. The Journal of the Acoustical Society of America, 21(4):419-428, 1949.

[10] B. Brouard, D. Lafarge, and J.F. Allard. A general method of modelling sound propagation in layered media. Journal of Sound and Vibration, 183(14):129-142, 1995.

[11] J. Brunskog. The influence of finite cavities on the sound insulation of doubleplate structures. The Journal of the Acoustical Society of America, 117(6):37273739, 2005.

[12] J. Brunskog and P. Davidsson. Sound transmission of structures; a finite element approach with simplified room description. Acta Acustica united with Acustica, 90(5):847-857, 2004. 
[13] J. Brunskog and P. Hammer. The interaction between the ISO tapping machine and lightweight floors. Acta Acustica united with Acustica, 89(2):296-308, 2003.

[14] J. Brunskog and P. Hammer. Prediction model for the impact sound level of lightweight floors. Acta Acustica united with Acustica, 89(2):309-322, 2003.

[15] Y.K. Cheung and S. Chakrabarti. Free vibration of thick, layered rectangular plates by a finite layer method. Journal of Sound and Vibration, 21(3):277-284, 1972.

[16] Y.K. Cheung and L.G. Tham. Finite strip method. CRC Press, 1997.

[17] H. Chung and G. Emms. Fourier series solutions to the vibration of rectangular lightweight floor / ceiling structures. Acta Acustica united with Acustica, 94(3):401-409, 2008.

[18] M.A. Delany and E.N. Bazley. Acoustic properties of fibrous absorbent materials. Applied Acoustics, 3(2):105-116, 1970.

[19] C. Díaz-Cereceda, J. Hetherington, J. Poblet-Puig, and A. Rodríguez-Ferran. A deterministic model of impact noise transmission through structural connections based on modal analysis. Journal of Sound and Vibration, 330(12):2801-2817, 2011.

[20] A. Dijckmans, G. Vermeir, and W. Lauriks. Sound transmission through finite lightweight multilayered structures with thin air layers. The Journal of the Acoustical Society of America, 128(6):3513-3524, 2010.

[21] F. Fahy. Sound and structural vibration. Academic Press, 1989.

[22] R. Friedrich. Finite strip method: 30 years. Engineering Computations, 17(1):92$111,2000$.

[23] L. Gagliardini, J. Roland, and J.L. Guyader. The use of a functional basis to calculate acoustic transmission between rooms. Journal of Sound and Vibration, 145(3):457-478, 1991.

[24] N. Geebelen, L. Boeckx, G. Vermeir, and W. Lauriks. A model for estimating the acoustic performances of multilayered systems based on a multiple extension of the transfer matrix method. In Proceedings of the Sixth European Conference on Noise Control, Tampere, Finland, 30 May - 1 June 2006, 2006.

[25] C. Guigou-Carter and M. Villot. Modelling of sound transmission through lightweight elements with stiffeners. Building Acoustics, 10(3):193-209, 2003.

[26] V. Hongisto. Sound insulation of double panels - comparison of existing prediction models. Acta Acustica united with Acustica, 92(1):61-78, 2006.

[27] C. Hopkins. Sound insulation. Elsevier Ltd., 2007. 
[28] U.E. Kernen and O.A.B. Hassan. Airborne sound insulation of a thin plate of finite dimensions. Acta Acustica united with Acustica, 91(4):732-739, 2005.

[29] W. Kropp and E. Rebillard. On the air-borne sound insulation of double wall constructions. Acta Acustica united with Acustica, 85(5):707-720, 1999.

[30] A. London. Transmission of reverberant sound through double walls. The Journal of the Acoustical Society of America, 22(2):270-279, 1950.

[31] Y. Miki. Acoustical properties of porous materials: modifications of DelanyBazley models. Journal of the Acoustical Society of Japan (E), 11(1):19-24, 1990.

[32] U. Orrenius and S. Finnveden. Calculation of wave propagation in rib-stiffened plate structures. Journal of Sound and Vibration, 198(2):203-224, 1996.

[33] R. Panneton and N. Atalla. Numerical prediction of sound transmission through finite multilayer systems with poroelastic materials. The Journal of the Acoustical Society of America, 100(1):346-354, 1996.

[34] J. Poblet-Puig and A. Rodríguez-Ferran. The finite strip method for acoustic and vibroacoustic problems. Journal of Computational Acoustics, 19(4):353-378, 2011.

[35] K. Renji, P.S. Nair, and S. Narayanan. On acoustic radiation resistance of plates. Journal of Sound and Vibration, 212(4):583-598, 1998.

[36] L.G. Sjökvist, J. Brunskog, and F. Jacobsen. Parameter survey of a rib stiffened wooden floor using sinus modes model. In Proceedings of the Acoustics'08 Conference, pages 3011-3015, Paris 2008.

[37] S. Smith, M. Allen, J. Puckett, and T. Edgar. The finite layer method for groundwater flow models. Water Resources Research, 28(6):1715-1722, 1992.

[38] A. Tadeu, J. António, and D. Mateus. Sound insulation provided by single and double panel walls - a comparison of analytical solutions versus experimental results. Applied Acoustics, 65(1):15-29, 2004.

[39] A. Trochidis and A. Kalaroutis. Sound transmission through double partitions with cavity absorption. Journal of Sound and Vibration, 107(2):321-327, 1986.

[40] T.E. Vigran. Building acoustics. Taylor and Francis, 2008.

[41] T.E. Vigran. Sound transmission in multilayered structures - introducing finite structural connections in the transfer matrix method. Applied Acoustics, 71(1):39-44, 2010.

[42] M. Villot, C. Guigou, and L. Gagliardini. Predicting the acoustical radiation of finite size multi-layered structures by applying spatial windowing on infinite structures. Journal of Sound and Vibration, 245(3):433-455, 2001. 
[43] J. Wang, T.J. Lu, J. Woodhouse, R.S. Langley, and J. Evans. Sound transmission through lightweight double-leaf partitions: theoretical modelling. Journal of Sound and Vibration, 286(4-5):817-847, 2005.

[44] E.G. Williams. A series expansion of the acoustic power radiated from planar sources. The Journal of the Acoustical Society of America, 73(5):1520-1524, 1983.

[45] F.X. Xin, T.J. Lu, and C.Q. Chen. Sound transmission through simply supported finite double-panel partitions with enclosed air cavity. Journal of Vibration and Acoustics, 132(1):011008, 2010.

[46] O.C. Zienkiewicz and R.L. Taylor. The finite element method. ButterworthHeinemann, 2000. 